\title{
Phase-type approximations perturbed by a heavy-tailed component for the Gerber-Shiu function of risk processes with two-sided jumps
}

\author{
Zbigniew Palmowski * \\ zbigniew.palmowski@gmail.com
}

\author{
Eleni Vatamidou ${ }^{\dagger}$ \\ eleni.vatamidou@unil.ch
}

\begin{abstract}
We consider in this paper a risk reserve process where the claims and gains arrive according to two independent Poisson processes. While the gain sizes are phase-type distributed, we assume instead that the claim sizes are phase-type perturbed by a heavy-tailed component; that is, the claim size distribution is formally chosen to be phase-type with large probability $1-\epsilon$ and heavy-tailed with small probability $\epsilon$. We analyze the seminal Gerber-Shiu function coding the joint distribution of the time to ruin, the surplus immediately before ruin, and the deficit at ruin. We derive its value as an expansion with respect to powers of $\epsilon$ with known coefficients and we construct approximations from the first two terms of the aforementioned series. The main idea is based on the so-called fluid embedding that allows to put the considered risk process into the framework of spectrally negative Markov-additive processes and use its fluctuation theory developed in [18].
\end{abstract}

Keywords: Gerber-Shiu function, Heavy-tailed claim sizes, Phase-type distribution, Fluctuation theory, Approximation, Perturbation

\section{Introduction}

Gerber and Shiu [13] investigated the classical insurance risk process

$$
X(t)=u+c t-\sum_{k=1}^{N(t)} U_{k}
$$

where $u \geq 0$ is the initial surplus, $c$ is the premium rate, $N(t), t \geq 0$, is a Poisson arrival process, and $\left\{U_{k}\right\}_{k=1}^{\infty}$ is a sequence of i.i.d. claim sizes independent of $N(t)$. For this process, they analyzed the function

$$
G S_{\omega}(u, q):=\mathbb{E}_{u}\left[e^{-q \tau} \omega(-X(\tau), X(\tau-)) \mathbb{1}_{\{\tau<\infty\}}\right]
$$

where

$$
\tau:=\inf \{t \geq 0: X(t)<0\}
$$

is the ruin time, $-X(\tau)$ is the deficit at ruin, $X(\tau-)$ is the surplus prior to ruin, and $\mathbb{E}_{u}[\cdot]=\mathbb{E}[\cdot \mid X(0)=u]$. The function $\omega:(0, \infty)^{2} \rightarrow[0, \infty)$ is any bounded, measurable penalty function, and $q \geq 0$ is the discount factor. Here the indicator function $\mathbb{1}_{\{\tau<\infty\}}$ emphasizes that the penalty is exercised only when ruin occurs. This function has found much attention since then and was given the name Gerber-Shiu (GS) or discounted penalty function.

\footnotetext{
*Faculty of Pure and Applied Mathematics, Wrocław University of Science and Technology, ul. Wyb. Wyspiańskiego 27, 50-370 Wrocław, Poland.

$\dagger$ The Faculty of Business and Economics, University of Lausanne, Quartier UNIL-Chamberonne Bâtiment Extranef, 1015 Lausanne, Switzerland.
} 
The Gerber-Shiu function has been widely used as an important risk measurement tool in the theory of bankruptcy, and at the same time, its existence triggered the research for many problems associated with bankruptcy. There are two main approaches allowing to identify the Gerber-Shiu function. The first one is based on solving appropriate (defective) renewal-type integro-differential equations, which is a consequence of the Markov property of the risk process and the fact that the GS function is harmonic with respect to its infinitesimal generator (see the discussion in [26]). The second approach is based on probabilistic methods, mainly related to martingale theory or the so-called scale functions in the context of Lévy-type risk processes (see [23] for details). In this paper, we choose the second method for analyzing the GS function.

Because of the importance of the GS function for risk theory, many authors have contributed to its analysis where the underlying risk reserve process has been generalized in several directions. The classical risk process (perturbed by Brownian motion) has been considered in [8, 13] (see also references therein). Later, Markov modulation (or regime switching) was added to the model; see [2] for a beautiful overview on this topic. The more general so-called spectrally negative Markov-additive risk process (MAP) was analyzed in [11]. Other interarrival times have also been considered. The Sparre Andersen risk process (perturbed by diffusion) with Erlang inter-arrival times was handled e.g. in [14, 25]. Some extensions have been considered lately. For example, a generalized discounted penalty function additionally takes into account the last minimum of the surplus before ruin in the analysis, see e.g. [5, 6].

This paper deals with another natural generalization of the classical risk process (1) that included occasional and random gains (apart from the constant premium intensity), which are modeled by additional positive jumps. That is, the risk process $X_{\epsilon}(t)$ takes the following form:

$$
X_{\epsilon}(t)=u+c t+\sum_{k=1}^{N^{(+)}(t)} U_{k}^{(+)}-\sum_{k=1}^{N_{\epsilon}^{(-)}(t)} U_{\epsilon, k}^{(-)} .
$$

We assume that $U_{k}^{(+)} \stackrel{d}{=} U^{(+)}$are i.i.d. random variables. Moreover, the claims $U_{\epsilon, k}^{(-)}$and gains $U_{k}^{(+)}$are independent of each other, where $\epsilon \in[0,1]$ is a parameter to be explained soon. Similarly, the gain arrival process $N^{(+)}(t)$ and the claim arrival process $N_{\epsilon}^{(-)}(t)$ do not depend on each other either.

Such models have already been investigated. For example, Xing et al. [30] studied the ruin time and the deficit at ruin for a risk process with double-sided jumps when the upward jumps are phase-type distributed and the downward jumps have an arbitrary distribution. Jacobsen [19] derived a similar result for a more general risk model, the Markov-modulated diffusion risk model with two-sided jumps. More recently, Zhang et al. [31] and Hua and Zaiming [15] studied a GS function for the classical risk process (4). They also studied the asymptotic estimate for the probability of ruin under heavy-tailed claims. Finally, Kolkovska and González [22] analyzed the risk process (4) perturbed by an $\alpha$-stable motion.

We combine another feature to our model: we include in our process some heavy-tailed risk in a quite specific way. We assume that our claims' umbrella consists of two kinds of claims, the so-called light-tailed (attritional) claims modeled by a phase-type distribution and the heavy-tailed (large) claims with some subexponential distribution. In daily practice, insurance companies often have a small amount of heavy-tailed risks in their portfolio in addition to those smaller ones. We model it by its proportion $\epsilon>0$. Thus, the general claim size distribution $F_{\epsilon}^{(-)}$can be expressed as follows:

$$
F_{\epsilon}^{(-)}:=(1-\epsilon) F_{p}+\epsilon F_{h}
$$

For simplicity, we assume that $F_{p}$ is of phase-type. Heavy-tailed risk is very dangerous for the insurance industry. Indeed, insurers quite often may become insolvent or experience financial distress due to large claims from catastrophic events and other large losses from financial investments. Such a study has become particularly relevant for insurance because of modern regulatory frameworks (e.g. EU Solvency) that require insurers to hold solvency capital so that the ruin probability is under control. 
Following [27] in this paper, we want to identify the impact of the heavy-tailed risk on the GS function, hence on the default probability, when its proportion $\epsilon$ tends to 0 , thus is very small. One can also treat this result as an approximation procedure for the GS function of the process (4) with phase-type upward gains and claims of the form (5). We manage to prove that

$$
G S_{\omega}^{\epsilon}(u, q)=G S_{\omega}(u, q)+\epsilon h(u, q)+O\left(\epsilon^{2}\right),
$$

where $h(u, q)$ can be found explicitly in terms of the model parameters.

The main idea is to use the so-called fluid embedding technique in which the upward jumps are placed at a slope one. This path transformation makes the risk process upward-continuous similarly to the classical risk process. Note that the original and transformed processes both get ruined over infinite time horizon or both survive but, obviously, this embedding affects the time scale. Since any upward jump has a phase-type distribution, which is the lifetime of some defective Markov chain, the modified risk process will move upwards with slope one as long as the Markov chain is still alive. Thus, we can treat any time of going upwards as the time of state changes of a certain Markov chain until its killing time. After this crucial path transformation, the risk process falls in the class of MAPs. We can then use the fluctuation theory for the latter developed in [18] based on scale matrices. As a next step, we expand these scale matrices with respect to $\epsilon$ and derive our main result.

The paper is organized as follows. In Section 2, we describe our model. Section 3 focuses on fluid embedding of upward jumps. In Section 4, we expand the scale matrices and other model parameters with respect to the above mentioned $\epsilon$. Section 5 gives the main result of this paper. Finally, we conclude in Section 6.

\section{Presentation of the model}

Let $X_{\epsilon}(t), t \geq 0$, be a stochastic process defined on the probability space $(\Omega, \mathcal{F}, \mathbb{P})$ that is equipped with a right-continuous complete filtration $\left\{\mathcal{F}_{t}\right\}_{t \geq 0}$. We assume that $X_{\epsilon}(t)$ represents the risk reserve process of an insurance company and we allow both for negative and positive claims, where the latter correspond to capital injections (gains). The gains $U^{(+)}$arrive according to a Poisson process $N^{(+)}$with rate $\lambda^{(+)}$and the claims $U_{\epsilon}^{(-)}$arrive according to a Poisson process $N_{\epsilon}^{(-)}$with rate $\lambda^{(-)}$. Finally, we assume that the positive safety loading condition $c+\lambda^{(+)} \mathbb{E} U^{(+)}-\lambda^{(-)} \mathbb{E} U_{\epsilon}^{(-)}>0$ holds. Thus, $X_{\epsilon}(t)$ takes the form (4), which was described briefly in Section 1.

In our model, we assume that $U_{k}^{(+)} \stackrel{d}{=} U^{(+)}$are i.i.d. random variables with jump size distribution $F^{(+)}$of phase-type with $m^{(+)}$phases and representation $\left(\boldsymbol{\alpha}^{(+)}, \mathbf{T}^{(+)}\right)$; see [2]. Moreover, the claims $U_{\epsilon, k}^{(-)} \stackrel{d}{=} U_{\epsilon}^{(-)}$are i.i.d. random variables with jump size distribution $F_{\epsilon}^{(-)}$. Motivated by statistical analysis, which proposes that only a small fraction of the upper-order statistics is relevant for estimating tail probabilities, we consider that an arbitrary claim size $U_{\epsilon}^{(-)}$is phase-type with probability $1-\epsilon$ and heavy-tailed with probability $\epsilon$, where $\epsilon \rightarrow 0$; see [10]. We assume that these phase-type claim sizes $B_{i} \stackrel{d}{=} B$ and the heavy-tailed claim sizes $C_{i} \stackrel{d}{=} C$ have both finite means, $\mu_{p}$ and $\mu_{h}$, respectively, are absolutely continuous, and we denote their corresponding distributions as $F_{p}$ and $F_{h}$.

Remark 2.1. Phase-type distributions are a computational vehicle for much of modern applied probability. Recall that a distribution $F$ is said to be of phase-type if it is the same distribution as the lifetime of some terminating Markov process starting from the vector $\boldsymbol{\alpha}$, having finitely many states, say $N$, and having time homogeneous transition rates with subintensity $\mathbf{T}$. Namely, $F(\phi)=1-\boldsymbol{\alpha} \exp \{\mathbf{T} \phi\} \mathbf{1}$, for $\phi \geq 0$, where $\mathbf{1}$ is a column-vector of ones. This gives also the form of the density $f(\phi)$ of $F$ as $f(\phi)=\boldsymbol{\alpha} \exp \{\mathbf{T} \phi\} \mathbf{t}$, where $\mathbf{t}=-\mathbf{T} \mathbf{1}$. Many well-known distributions are of phase-type; for example, the exponential distribution with intensity $\rho$ is $\mathrm{PH}$ in this case with $N=1, \mathbf{T}=\rho$, and $\boldsymbol{\alpha}=1$. Other more involved distributions that belong to the class of phase-types are the hyperexponential, the Erlang, and the Coxian; see [2] for a detailed description. 


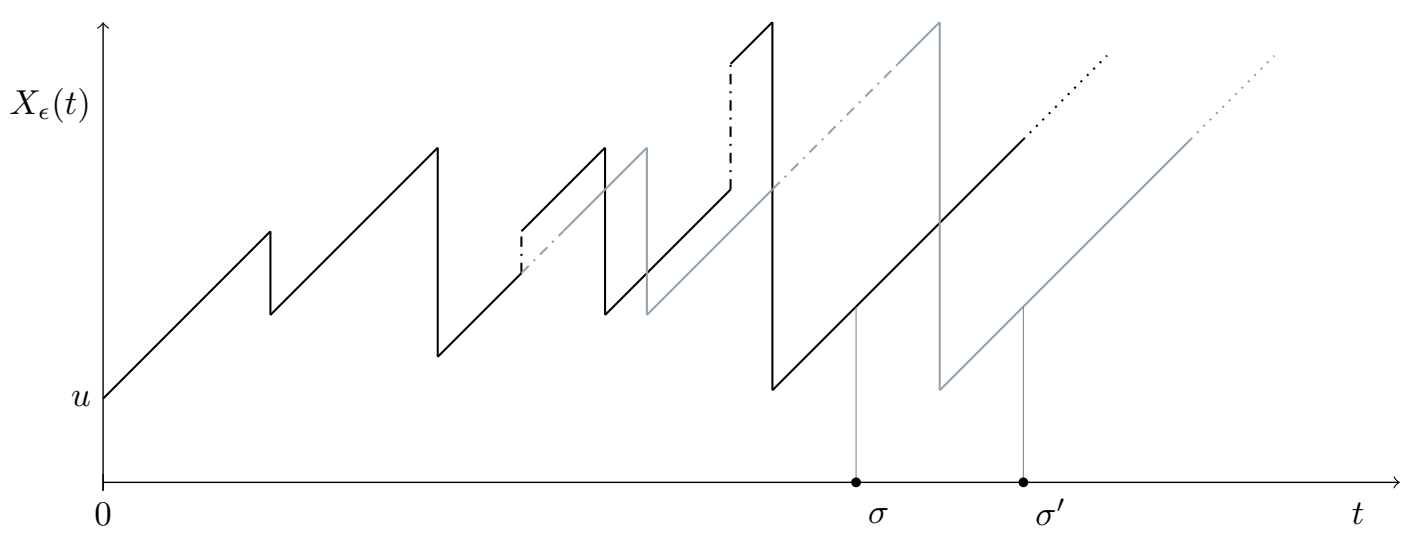

Figure 1: Sample path of a MAP with two-sided jumps (black line), where the positive phase-type jumps (dashed-dotted line) are replaced by linear stretches of slope 1, resulting in a spectrally-negative MAP with an augmented state-space (gray line). Fluid embedding shifts time $\sigma$ in the initial MAP to $\sigma^{\prime}$ in the spectrally-negative MAP.

For the above mentioned model, we are interested in evaluating the so-called Gerber-Shiu or expected discounted penalty function. If the time to ruin is defined as $\tau_{\epsilon}:=\inf \left\{t \geq 0: X_{\epsilon}(t)<0\right\}$, then we have the following definition from [13].

Definition 2.2. The Gerber-Shiu or expected discounted penalty function is defined as

$$
G S_{\omega}^{\epsilon}(u, q):=\mathbb{E}_{u}\left[e^{-q \tau_{\epsilon}} \omega\left(-X_{\epsilon}\left(\tau_{\epsilon}\right), X_{\epsilon}\left(\tau_{\epsilon}-\right)\right) \mathbb{1}_{\left\{\tau_{\epsilon}<\infty\right\}}\right]
$$

where $-X_{\epsilon}\left(\tau_{\epsilon}\right)$ is the deficit at ruin, $X_{\epsilon}\left(\tau_{\epsilon}-\right)$ is the surplus prior to ruin, and $u \geq 0$ is the initial surplus. The function $\omega:(0, \infty)^{2} \rightarrow[0, \infty)$ is any bounded, measurable function, and $q \geq 0$, is the discount factor. Here the indicator function $\mathbb{1}_{\left\{\tau_{\epsilon}<\infty\right\}}$ emphasizes that the penalty is exercised only when ruin occurs.

Assume from now on that $q>0$. In the absence of positive jumps, the model belongs to the class of spectrally negative Lévy-processes, i.e. it is a stochastic process with càdlàg (right-continuous with left limits) sample paths, and stationary and independent increments, and the Gerber-Shiu function takes the form [23, p.43]

$$
G S_{\omega}^{\epsilon}(u, q)=\lambda^{(-)} \int_{[0, \infty)} \int_{[0, \infty)} \omega(y, z) r^{(q)}(u, z) F_{\epsilon}^{(-)}(z+d y) d z
$$

where $r^{(q)}(u, z)$ is known as the $q$-resolvent or potential measure of $X_{\epsilon}(t)$ killed on exiting the positive half-line. In the case of existing upward jumps, we have to place our risk process into the framework of spectrally negative MAPs via fluid embedding. The spectral negativity means that our MAP has no positive jumps. In this way, we construct a new process $Y_{\epsilon}(t)$ governed by an environmental process $J_{\epsilon}(t)$ for which we will apply an identity similar to Equation (7). We explain in the next section how to construct an equivalent spectrally negative MAP and we summarize the respective necessary results for the evaluation of the Gerber-Shiu function.

\section{Fluid embedding and the Gerber-Shiu function for MAPs}

When in a model the positive jumps are phase-type, a common approach is to spread them out as a succession of linear pieces of unit slope as it was described e.g. in [4, 7]; see Figure 1. This procedure, which is called fluid embedding, requires adding supplementary states to the background process as many as the phases; namely $m^{(+)}$in our model. By applying this technique, we construct an auxiliary spectrally negative MAP $Y_{\epsilon}(t)$ with an enlarged state space, say $E=\left\{1,2, \ldots, m^{(+)}+1\right\}$. The 1 st state corresponds to the original risk process with negative jumps only. The other states correspond to the upward jumps transformed into lines. For the latter states, we have $d Y_{\epsilon}(t)=d t$. In addition, we denote by $J_{\epsilon}(t)$ the right-continuous jump process that lives on $E$. 
Note that for the process in Equation (4), jumps are coming according to a superposition of the two independent Poisson processes $N^{(+)}(t)$ and $N_{\epsilon}^{(-)}(t)$. Hence, we obtain Equation (7) from the compensation formula (see [23, Theorem 3.4]) because ruin can occur only when $N_{\epsilon}^{(-)}(t)$ increases, i.e. when a claim arrives, and thus only $\lambda^{(-)}$and $F_{\epsilon}^{(-)}$appear in the equation. In addition, we know from [23, p.39] that $r^{(q)}(u, z)=\frac{1}{q d z} \mathbb{P}_{u}\left(X_{\epsilon}\left(e_{q}\right) \in d z, \inf _{w \leq e_{q}} X_{\epsilon}(w) \geq 0\right)$, where $e_{q}$ is an exponential random variable with parameter $q>0$ and independent of $X_{\epsilon}(t)$, while $\mathbb{P}_{u}(\cdot)=\mathbb{P}\left(\cdot \mid X_{\epsilon}(0)=u\right)$. Note that for fixed $u$, the resolvent $r^{(q)}(u, z)$ should be treated as a Radon-Nikodym derivative of $\frac{1}{q d z} \mathbb{P}_{u}\left(X_{\epsilon}\left(e_{q}\right) \in d z, \inf _{w \leq e_{q}} X_{\epsilon}(w) \geq 0\right)$ with respect to the Lebesgue measure. Let now $\mathbf{r}_{\epsilon}^{(q)}(u, z)$ be the $q$-resolvent of the process $\left(Y_{\epsilon}(t), J_{\epsilon}(t)\right)$ constructed with fluid embedding killed on exiting from the positive half-line, i.e.

$$
\mathbf{r}_{\epsilon}^{(q)}(u, z):=\int_{0}^{\infty} e^{-q t} \mathbb{P}_{u}\left(Y_{\epsilon}(t) \in d z, \inf _{w \leq t} Y_{\epsilon}(w) \geq 0, J_{\epsilon}(t)\right) d t / d z,
$$

where for a random variable $L$ we write $\mathbb{E}_{u}\left[L ; J_{\epsilon}(t)\right]$ to denote a matrix with $i j$-th element

$$
\mathbb{E}_{u, i}\left[L ; J_{\epsilon}(t)=j\right]=\mathbb{E}\left[L \mathbb{1}_{\left\{J_{\epsilon}(t)=j\right\}} \mid Y_{\epsilon}(0)=u, J_{\epsilon}(0)=i\right],
$$

and $\mathbb{P}_{u}\left(A, J_{\epsilon}(t)\right)=\mathbb{E}_{u}\left[\mathbb{1}_{A} ; J_{\epsilon}(t)\right]$; see also [11]. Observe that all states in $E$ are fictitious for $X_{\epsilon}(t)$ except for state 1 . Moreover, stopping the process $X_{\epsilon}\left(e_{q}\right)$ at the exponential clock is possible only when this process has no jump at that moment $e_{q}$. That is, in particular $e_{q}$ cannot happen at the moment of a positive jump. Thus, if we want to stop the process $Y_{\epsilon}(t)$ at an exponential epoch $e_{q}$ at the same level as $X_{\epsilon}\left(e_{q}\right)$, then we should condition on the event that the state $J_{\epsilon}\left(e_{q}\right)$ is equal to 1 , since we take into account only the scenarios of stopping that happen at state 1 . As a result, if $A(q)=1 / \mathbb{P}_{u, 1}\left(J_{\epsilon}\left(e_{q}\right)=1\right)$, it follows that $r^{(q)}(u, z)=\frac{1}{q d z} \mathbb{P}_{u, 1}\left(Y_{\epsilon}\left(e_{q}\right) \in d z \mid J_{\epsilon}\left(e_{q}\right)=1\right)=A(q) \mathbf{r}_{\epsilon}^{(q)}(u, z)_{(1,1)}$ due to the lack of memory of the exponential distribution. Consequently, the GS function takes the next form after fluid embedding

$$
G S_{\omega}^{\epsilon}(u, q)=A(q) \lambda^{(-)} \int_{[0, \infty)} \int_{[0, \infty)} \omega(y, z) \mathbf{r}_{\epsilon}^{(q)}(u, z)_{(1,1)} F_{\epsilon}^{(-)}(z+d y) d z
$$

Recall that the process $\left(Y_{\epsilon}(t), J_{\epsilon}(t)\right)$ is a MAP if the pair $\left(Y_{\epsilon}(t+s)-Y_{\epsilon}(t), J_{\epsilon}(t+s)\right)$ given $\left\{J_{\epsilon}(t)=i\right\}$ is independent of the natural history $\mathcal{F}_{t}$ up to time $t$ and has the same law as $\left(Y_{\epsilon}(s)-Y_{\epsilon}(0), J_{\epsilon}(s)\right)$ given $\left\{J_{\epsilon}(0)=i\right\}$, for all $s, t \geq 0$ and $i \in E$. It is common to say that $Y_{\epsilon}(t)$ is an additive component and $J_{\epsilon}(t)$ is a background process representing the environment. Importantly, MAPs have a very special structure, which we reveal in the following. It is immediate that $J_{\epsilon}(t)$ is a Markov chain. Furthermore, $Y_{\epsilon}(t)$ evolves as some Lévy process $X_{\epsilon, i}(t)$ while $J_{\epsilon}(t)=i$. In addition, a transition of $J_{\epsilon}(t)$ from $i$ to $j \neq i$ triggers a jump of $Y_{\epsilon}(t)$. All the above components are independent.

Since $Y_{\epsilon}(t)$ has no positive jumps, it is uniquely characterized by the matrix exponent $\mathbf{F}_{\epsilon}(s), s \geq 0$, which satisfies

$$
\mathbb{E}\left[e^{s Y_{\epsilon}(t)} ; J_{\epsilon}(t)\right]=e^{\mathbf{F}_{\epsilon}(s) t}, \quad t \geq 0 .
$$

See [2] for all details. If $\tilde{F}_{p}^{e}(s)$ and $\tilde{F}_{h}^{e}(s)$ are the Laplace-Stieltjes transforms (LSTs) of the stationary-excess claim size distributions $F_{p}^{e}(x)$ and $F_{h}^{e}(x)$ ( $B^{e}$ and $C^{e}$ are the corresponding stationary-excess claim sizes), respectively, then from the above described fluid embedding, it follows that the matrix exponent $\mathbf{F}_{\epsilon}(s)$ takes the form

$$
\mathbf{F}_{\epsilon}(s)=\left[\begin{array}{c|c}
\phi_{\epsilon}(s)-\lambda^{(+)} & \lambda^{(+)} \boldsymbol{\alpha}^{(+)} \\
\hline \mathbf{t}^{(+)} & \mathbf{T}^{(+)}+s \mathbf{I}
\end{array}\right],
$$

where

$$
\phi_{\epsilon}(s)=c s-\lambda^{(-)} s\left((1-\epsilon) \mu_{p} \tilde{F}_{p}^{e}(s)+\epsilon \mu_{h} \tilde{F}_{h}^{e}(s)\right),
$$

and $\mathbf{I}$ is the identity matrix of appropriate dimension. Note that $A(q)=\left[q\left(q \mathbf{I}-\mathbf{F}_{\epsilon}(0)\right)_{(1,1)}^{-1}\right]^{-1}$. 
Remark 3.1. Observe that $\phi_{\epsilon}(s)$ corresponds to the Lévy exponent of $X_{\epsilon}(t)$ in case the rate of the capital injections $\lambda^{(+)}$is equal to zero; namely when there exist only negative jumps. Moreover, note that $\mathbf{F}_{\epsilon}(0)$ is the transition rate matrix of $J_{\epsilon}(t)$.

From Equation (9), it is clear that we need to evaluate the $q$-resolvent measure for $Y_{\epsilon}(t)$, which is known to be expressed in terms of the so-called $q$-scale matrices [18]. For this reason, we provide first its formal definition.

Proposition 3.2. There exists a unique continuous function $\mathbf{W}_{\epsilon}^{(q)}:[0, \infty) \rightarrow \mathbb{R}^{\left(m^{(+)}+1\right) \times\left(m^{(+)}+1\right)}$, such that $\mathbf{W}_{\epsilon}^{(q)}(x)$ is invertible for all $x>0$,

$$
\int_{0}^{\infty} e^{-s x} \mathbf{W}_{\epsilon}^{(q)}(x) d x=\left(\mathbf{F}_{\epsilon}(s)-\mathcal{Q}\right)^{-1}
$$

for all $s>\eta(q)=\max \left\{\Re(z): z \in \mathbb{C}, \operatorname{det}\left(\mathbf{F}_{\epsilon}(s)-\mathcal{Q}\right)=0\right\}$, where $\mathcal{Q}=\operatorname{diag}\{q, 0, \ldots, 0\}$.

Proof. Following [7], we do not discount the time for the states $2, \ldots, m^{(+)}+1$ in order to account for the jump nature of the new phase-type upward movements. The result is then a direct application of [18, Theorem 1].

For simplicity, we set $\mathbf{F}_{\epsilon}^{(q)}(s)=\mathbf{F}_{\epsilon}(s)-\mathcal{Q}$. By using Theorem 4.8 of [16], one can immediately deduct that the equation $\operatorname{det} \mathbf{F}_{\epsilon}^{(q)}(s)=0$ has $m^{(+)}+1$ zeros with positive real part if $q>0$. Contrarily, for $q=0$ the number of zeros in $\mathbb{C}^{\Re>0}$ are in number $m^{(+)}$and zero is also a root. The latter is obvious since the first column of matrix $\mathbf{F}_{\epsilon}(0)$ is a linear combination of all its remaining columns. The solutions of $\operatorname{det} \mathbf{F}_{\epsilon}^{(q)}(s)=0$ are called eigenvalues of the matrix $\mathbf{F}_{\epsilon}^{(q)}(s)$. We assume here that these eigenvalues are simple; namely their multiplicities are all equal to one.

Following [11, 17], we define the matrix $\mathbf{R}_{\epsilon}^{(q)}$ as the unique left solution to the matrix equation $\mathbf{F}_{\epsilon}^{(q)}\left(\mathbf{R}_{\epsilon}^{(q)}\right)=\mathbb{O}$. This matrix is positive stable, i.e. all its eigenvalues have positive real part, and it is also diagonizable. More details regarding its construction will be provided in Section 4.3.

In the next lemma, we provide the exact expression for the $q$-resolvent measure of $Y_{\epsilon}(t)$.

Lemma 3.3. Fix $q>0$. The $q$-resolvent measure for $Y_{\epsilon}(t)$ killed on exiting $[0, \infty)$ has density given by

$$
\mathbf{r}_{\epsilon}^{(q)}(u, z)=\mathbf{W}_{\epsilon}^{(q)}(u) e^{-\mathbf{R}_{\epsilon}^{(q)} z}-\mathbf{W}_{\epsilon}^{(q)}(u-z)
$$

for all $u, z \geq 0$.

Proof. The result is immediate from [17, Theorem 1], by taking in Equation (14) $a=u$ and $x=z-u$, since we have here $X_{\epsilon}(0)=u$. The above resolvent identity appears also in [11].

A direct consequence of Lemma 3.3 is that the GS function in Equation (9) further simplifies to

$$
G S_{\omega}^{\epsilon}(u, q)=A(q) \lambda^{(-)} \int_{[0, \infty)} \int_{[0, \infty)} \omega(y, z)\left(\mathbf{W}_{\epsilon}^{(q)}(u) e^{-\mathbf{R}_{\epsilon}^{(q)} z}-\mathbf{W}_{\epsilon}^{(q)}(u-z)\right)_{(1,1)} F_{\epsilon}^{(-)}(z+d y) d z .
$$

Although the computational efforts have simplified significantly, it is still not easy to find a closed-form expression for the Gerber-Shiu function in Equation (15). The problem arises from the fact that $\tilde{F}_{h}^{e}(s)$, which is the Laplace transform of a heavy-tailed distribution, appears in every element of the matrix $\left(\mathbf{F}_{\epsilon}(s)-\mathcal{Q}\right)^{-1}$ (specifically at the denominator, but we omit the details at this point), thus making Laplace inversion to evaluate the scale matrix difficult, if not impossible. However, if $\tilde{F}_{h}^{e}(s)$ was missing, then $\left(\mathbf{F}_{\epsilon}(s)-\mathcal{Q}\right)^{-1}$ would be a matrix of rational functions in $s$, and the evaluation of the scale matrix would be straightforward. Using this key idea, we explain in the next section how we can exploit that the claim size distribution is a mixture of $F_{p}$ and $F_{h}$ and apply perturbation analysis to derive a series expansion for the Gerber-Shiu function. The goal is then to use the series expansion and construct accurate approximations for the measure under consideration. 


\section{Perturbation of the model parameters}

Recall that the claim size distribution $F_{\epsilon}^{(-)}$is a mixture of a phase-type distribution $F_{p}$ and a heavy-tailed one $F_{h}$, i.e. $F_{\epsilon}^{(-)}(x)=(1-\epsilon) F_{p}(x)+\epsilon F_{h}(x), x \geq 0$. As mentioned in the previous section, if there were not any heavy-tailed jumps, for example if $F_{\epsilon}^{(-)}(x)=F_{p}(x), x \geq 0$, then the algebraic calculations would reduce to the Laplace inversion of rational functions. Now, notice that

$$
F_{\epsilon}^{(-)}(x)=(1-\epsilon) F_{p}(x)+\epsilon F_{h}(x)=F_{p}(x)+\epsilon\left(F_{h}(x)-F_{p}(x)\right),
$$

which means that the claim size distribution $F_{\epsilon}^{(-)}$can be regarded as perturbation of the distribution $F_{p}(x)$ by the function $\epsilon\left(F_{h}(x)-F_{p}(x)\right)$. Consequently, we could calculate the Gerber-Shiu function with the claim size distribution $F_{p}(x)$ and then use perturbation analysis in order to derive a series expansion (or first order approximation) in $\epsilon$ for $G S_{\omega}^{\epsilon}(u, q)$.

Observe that perturbation on the claim size distribution affects also the scale matrix $\mathbf{W}_{\epsilon}^{(q)}(u)$ as well as the matrix $\mathbf{R}_{\epsilon}^{(q)}$. Therefore, we first introduce the notation for the model with claim size distribution $F_{p}(x)$, which is obviously our phase-type base model, and then explain how to derive the parameters of the original model as perturbation of the parameters of the phase-type base model.

\subsection{Phase-type base model}

Setting $\epsilon=0$ in Equation (16) allows us to retrieve $F_{\epsilon}^{(-)}(x)=F_{p}(x)$. As we can see, the resulting mixture $F_{\epsilon}^{(-)}(x)$ is independent of the parameter $\epsilon$, and so are all the other parameters of this base model. Thus, to avoid overloading the notation, we omit the subscript "0" (which is a consequence of the fact that $\epsilon=0$ ) from the parameters of the phase-type base model, which we call base model for simplicity. The Laplace exponent of the base model is

$$
\mathbf{F}^{(q)}(s)=\left[\begin{array}{c|c}
\phi(s)-\lambda^{(+)}-q & \lambda^{(+)} \boldsymbol{\alpha}^{(+)} \\
\hline \mathbf{t}^{(+)} & \mathbf{T}^{(+)}+s \mathbf{I}
\end{array}\right],
$$

where

$$
\phi(s)=c s-\lambda^{(-)} s \mu_{p} \tilde{F}_{p}^{e}(s) .
$$

Setting $k(s)=\lambda^{(-)}\left(\mu_{h} \tilde{F}_{h}^{e}(s)-\mu_{p} \tilde{F}_{p}^{e}(s)\right)$ for simplicity, we observe that $\phi_{\epsilon}(s)=\phi(s)-\epsilon s k(s)$ and that $\mathbf{F}_{\epsilon}^{(q)}(s)=$ $\mathbf{F}^{(q)}(s)-\epsilon \mathbf{K}(s)$, where

$$
\mathbf{K}(s)=\left[\begin{array}{c|c}
s k(s) & \mathbf{0} \\
\hline \mathbf{0} & \mathbf{0}
\end{array}\right] .
$$

In the following sections, we explain how to find the scale matrix $\mathbf{W}_{\epsilon}^{(q)}(x)$ and the matrix $\mathbf{R}_{\epsilon}^{(q)}$ as perturbation of the corresponding matrices $\mathbf{W}^{(q)}(x)$ and $\mathbf{R}^{(q)}$ in the base model. We start with $\mathbf{W}_{\epsilon}^{(q)}(x)$.

\subsection{The perturbed scale matrix}

According to Equation (13), the scale matrix $\mathbf{W}_{\epsilon}^{(q)}(x)$ is defined as the Laplace inversion of the matrix $\left(\mathbf{F}_{\epsilon}^{(q)}(s)\right)^{-1}$, the series expansion of which is given in the following line:

$$
\left(\mathbf{F}_{\epsilon}^{(q)}(s)\right)^{-1}=\left(\mathbf{F}^{(q)}(s)-\epsilon \mathbf{K}(s)\right)^{-1}=\sum_{n=0}^{\infty} \epsilon^{n}\left(\left(\mathbf{F}^{(q)}(s)\right)^{-1} \mathbf{K}(s)\right)^{n}\left(\mathbf{F}^{(q)}(s)\right)^{-1} .
$$

Note that the matrix $\mathbf{K}(s)$ is everywhere 0 except for its $(1,1)$ element. Thus, only the 1st row and 1st column of $\left(\mathbf{F}^{(q)}(s)\right)^{-1}$ are involved in the series expansion (20). If $\mathfrak{F}^{(q)}(s)$ is the adjoint matrix of $\mathbf{F}^{(q)}(s)$, then it is known that $\left(\mathbf{F}^{(q)}(s)\right)^{-1}=\mathfrak{F}^{(q)}(s) / \operatorname{det} \mathbf{F}^{(q)}(s)$. Let $\mathfrak{f}_{i j}^{(q)}$ be the $(i, j)$ element of the matrix $\mathfrak{F}^{(q)}(s)$. Hence 
Equation (20) takes the form

$$
\begin{aligned}
\left(\mathbf{F}_{\epsilon}^{(q)}(s)\right)^{-1}= & \left(\mathbf{F}^{(q)}(s)\right)^{-1}+\sum_{n=1}^{\infty} \epsilon^{n} s^{n} k^{n}(s) \frac{\left(\operatorname{det}\left(\mathbf{T}^{(+)}+s \mathbf{I}\right)\right)^{n-1}}{\left(\operatorname{det} \mathbf{F}^{(q)}(s)\right)^{n+1}} \\
& \times\left(\begin{array}{cccc}
\mathfrak{f}_{11}^{(q)} \mathfrak{f}_{11}^{(q)} & \mathfrak{f}_{11}^{(q)} \mathfrak{f}_{12}^{(q)} & \cdots & \mathfrak{f}_{11}^{(q)} \mathfrak{f}_{1, m}^{(q)}(+)+1 \\
\mathfrak{f}_{21}^{(q)} \mathfrak{f}_{11}^{(q)} & \mathfrak{f}_{21}^{(q)} \mathfrak{f}_{12}^{(q)} & \cdots & \mathfrak{f}_{21}^{(q)} \mathfrak{f}_{1, m}^{(q)}(+)+1 \\
\vdots & \vdots & \ddots & \vdots \\
\mathfrak{f}_{m(+)+1,1}^{(q)} \mathfrak{f}_{11}^{(q)} & \mathfrak{f}_{m(+)+1,1}^{(q)} \mathfrak{f}_{12}^{(q)} & \cdots & \mathfrak{f}_{m(+)+1,1}^{(q)} \mathfrak{f}_{1, m(+)+1}^{(q)}
\end{array}\right),
\end{aligned}
$$

where $\mathfrak{f}_{11}^{(q)}=\operatorname{det}\left(\mathbf{T}^{(+)}+s \mathbf{I}\right)$. Recall now that we only need the $(1,1)$ element of the matrix $\mathbf{r}_{\epsilon}^{(q)}(u, z)$. Therefore, according to Equation (14), we just need to find the Laplace inversion of the first row of the matrix exponent $\left(\mathbf{F}_{\epsilon}^{(q)}(s)\right)^{-1}$. In particular, its $(1,1)$ element has the series representation

$$
\left(\mathbf{F}_{\epsilon}^{(q)}(s)\right)_{(1,1)}^{-1}=\left(\mathbf{F}^{(q)}(s)\right)_{(1,1)}^{-1}+\sum_{n=1}^{\infty} \epsilon^{n} k^{n}(s)\left(\frac{s \operatorname{det}\left(\mathbf{T}^{(+)}+s \mathbf{I}\right)}{\operatorname{det} \mathbf{F}^{(q)}(s)}\right)^{n}\left(\mathbf{F}^{(q)}(s)\right)_{(1,1)}^{-1},
$$

given that $\left(\mathbf{F}^{(q)}(s)\right)_{(1,1)}^{-1}=\operatorname{det}\left(\mathbf{T}^{(+)}+s \mathbf{I}\right) / \operatorname{det} \mathbf{F}^{(q)}(s)$.

From [18, Theorem 5] and the subsequent comment, it follows that the scale matrices $\mathbf{W}_{\epsilon}^{(q)}(x)$ and $\mathbf{W}^{(q)}(x)$ are entry-wise differentiable. Hence, by following the reasoning in [23, Section 4.1], we let $\mathbf{W}_{\epsilon}^{(q)}(d x)$ and $\mathbf{W}^{(q)}(d x)$ be the matrices such that $\int_{0}^{\infty} e^{-s x} \mathbf{W}_{\epsilon}^{(q)}(d x)=s\left(\mathbf{F}_{\epsilon}^{(q)}(s)\right)^{-1}$ and $\int_{0}^{\infty} e^{-s x} \mathbf{W}^{(q)}(d x)=s\left(\mathbf{F}^{(q)}(s)\right)^{-1}$, respectively. Moreover, taking into account the binomial identity $k(s)=\left(\lambda^{(-)}\right)^{n} \sum_{m=0}^{n}\left(\begin{array}{c}n \\ m\end{array}\right) \mu_{h}^{n-m}\left(-\mu_{p}\right)^{m}\left(\tilde{F}_{h}^{e}(s)\right)^{n-m}\left(\tilde{F}_{p}^{e}(s)\right)^{m}$, we apply Laplace inversion to Equation (22) to obtain

$$
\begin{aligned}
\left(\mathbf{W}_{\epsilon}^{(q)}(d x)\right)_{(1,1)}= & \left(\mathbf{W}^{(q)}(d x)\right)_{(1,1)} \\
& +\sum_{n=1}^{\infty} \epsilon^{n}\left(\lambda^{(-)}\right)^{n} \sum_{m=0}^{n}\left(\begin{array}{c}
n \\
m
\end{array}\right) \mu_{h}^{n-m}\left(-\mu_{p}\right)^{m} L_{n-m, m}(d x) *\left(\mathbf{W}^{(q)}(d x)\right)_{(1,1)}^{*(n+1)},
\end{aligned}
$$

where $B_{i}^{e} \stackrel{d}{=} B^{e}$ and $C_{i}^{e} \stackrel{d}{=} C^{e}$ are respectively the stationary-excess phase-type and heavy-tailed claim sizes, $L_{s, r}(d x)=\mathbb{P}\left(C_{1}^{e}+\cdots+C_{s}^{e}+B_{1}^{e}+\cdots+B_{r}^{e} \in d x\right)$, the symbol $*$ denotes the convolution between functions, and $\left(\mathbf{W}^{(q)}(d x)\right)_{(1,1)}^{* n}$ is the $n$th convolution of $\left(\mathbf{W}^{(q)}(d x)\right)_{(1,1)}$ with itself. Any other element of the first row of the matrix expansion (21) takes the form

$$
\left(\mathbf{F}_{\epsilon}^{(q)}(s)\right)_{(1, j)}^{-1}=\left(\mathbf{F}^{(q)}(s)\right)_{(1, j)}^{-1}+\sum_{n=1}^{\infty} \epsilon^{n} k^{n}(s)\left(\frac{s \operatorname{det}\left(\mathbf{T}^{(+)}+s \mathbf{I}\right)}{\operatorname{det} \mathbf{F}^{(q)}(s)}\right)^{n}\left(\mathbf{F}^{(q)}(s)\right)_{(1, j)}^{-1},
$$

and consequently, Laplace inversion gives

$$
\begin{aligned}
\left(\mathbf{W}_{\epsilon}^{(q)}(d x)\right)_{(1, j)} & =\left(\mathbf{W}^{(q)}(d x)\right)_{(1, j)} \\
& +\sum_{n=1}^{\infty} \epsilon^{n}\left(\lambda^{(-)}\right)^{n} \sum_{m=0}^{n}\left(\begin{array}{c}
n \\
m
\end{array}\right) \mu_{h}^{n-m}\left(-\mu_{p}\right)^{m} L_{n-m, m}(d x) *\left(\mathbf{W}^{(q)}(d x)\right)_{(1,1)}^{* n} *\left(\mathbf{W}^{(q)}(d x)\right)_{(1, j)}
\end{aligned}
$$

Given Equations (23) and (25), it is easy to find the elements of the scale matrix $\mathbf{W}_{\epsilon}^{(q)}(x)$ that are important for the evaluation of the Gerber-Shiu function. In the next section, we turn our attention to the matrix $\mathbf{R}_{\epsilon}^{(q)}$, which relates to the eigenvalues with non-negative real part (and their eigenvectors) of $\mathbf{F}_{\epsilon}^{(q)}(s)$.

Remark 4.1. Observe that we denote by $f(x) * g(x)$ the convolution between the functions $f$ and $g$ instead of the traditional choice $(f * g)(x)$, i.e. $f(x) * g(x)=\int_{0}^{x} f(t) g(x-t) d t$. This convention is used in order to avoid the introduction of new notation, e.g. it allows us to use interchangeably the convolutions $L_{n-m, m}(x) * \mathbf{W}^{(q)}(d x)$ 
and $L_{n-m, m}(d x) * \mathbf{W}^{(q)}(x)$, while the same principle applies to convolutions of similar form.

\subsection{The perturbed matrix $\mathbf{R}_{\epsilon}^{(q)}$}

Since $\mathbf{R}_{\epsilon}^{(q)}$ is diagonizable, this means that $\mathbf{R}_{\epsilon}^{(q)}=\left(\mathbf{L}_{\epsilon}^{(q)}\right)^{-1} \boldsymbol{\Lambda}_{\epsilon}^{(q)} \mathbf{L}_{\epsilon}^{(q)}$ for some invertible matrix $\mathbf{L}_{\epsilon}^{(q)}$ and a real diagonal matrix $\boldsymbol{\Lambda}_{\epsilon}^{(q)}$. More precisely, the diagonal elements of $\boldsymbol{\Lambda}_{\epsilon}^{(q)}$ are exactly the $m^{(+)}+1$ solutions of $\operatorname{det} \mathbf{F}_{\epsilon}^{(q)}(s)=0$ with non-negative real part; let us denote them as $\rho_{\epsilon, i}^{(q)}, i=1, \ldots, m^{(+)}+1$ (we order them w.l.o.g. according to their real part). Then, with similar arguments as in [16, Corollary 3.6], it can easily be shown that the $i$ th row of $\mathbf{L}_{\epsilon}^{(q)}$ belongs to the (left) nullspace of $\mathbf{F}_{\epsilon}^{(q)}\left(\rho_{\epsilon, i}^{(q)}\right), i \in E$.

Thus, in order to construct the matrix $\mathbf{R}_{\epsilon}^{(q)}$, we need to find first the eigenvalues of $\mathbf{F}_{\epsilon}^{(q)}(s)$, i.e. the solutions of the equation $\operatorname{det} \mathbf{F}_{\epsilon}^{(q)}(s)=0$ with non-negative real part. We calculate these eigenvalues in Section 4.3.1. Afterwards, in Section 4.3.2, we explain how to construct the matrix $\mathbf{L}_{\epsilon}^{(q)}$.

\subsubsection{The perturbed eigenvalues with positive real part}

We can easily calculate

$$
\operatorname{det} \mathbf{F}_{\epsilon}^{(q)}(s)=\operatorname{det} \mathbf{F}^{(q)}(s)-\epsilon s k(s) \operatorname{det}\left(\mathbf{T}^{(+)}+s \mathbf{I}\right),
$$

and it is rather obvious that 0 is an eigenvalue (simple) of $\mathbf{F}_{\epsilon}^{(q)}(s)$ only when $q=0$, which is excluded for now. Let now $\rho_{i}^{(q)}$ be any other simple eigenvalue of $\mathbf{F}^{(q)}(s)$ with positive real part. Assuming that both $\mathbf{F}^{(q)}(s)$ and $\mathbf{K}(s)$ are analytic in the neighbourhood of $\rho_{i}^{(q)}$, it is known from [21, Chapter Two §1] that there exists a unique eigenvalue $\rho_{\epsilon, i}^{(q)}$ of $\mathbf{F}_{\epsilon}^{(q)}(s)$ with expansion

$$
\rho_{\epsilon, i}^{(q)}=\rho_{i}^{(q)}+\epsilon \delta_{i, 1}^{(q)}+\epsilon^{2} \delta_{i, 2}^{(q)}+\ldots
$$

Thus, if $\boldsymbol{\Lambda}^{(q)}=\operatorname{diag}\left(\rho_{i}^{(q)}\right)_{i \in E}$, then $\boldsymbol{\Lambda}_{\epsilon}^{(q)}$ can be written as perturbation of $\boldsymbol{\Lambda}^{(q)}$ as follows:

$$
\boldsymbol{\Lambda}_{\epsilon}^{(q)}=\boldsymbol{\Lambda}^{(q)}+\epsilon \boldsymbol{\Delta}_{1}^{(q)}+\epsilon^{2} \boldsymbol{\Delta}_{2}^{(q)}+\ldots
$$

Remark 4.2. The exact value of the coefficient $\delta_{i, 1}^{(q)}$ has already been evaluated in [28, Corollary A.11] and it is equal to

$$
\delta_{i, 1}^{(q)}=\frac{k\left(\rho_{i}^{(q)}\right) \operatorname{det}\left(\mathbf{T}^{(+)}+\rho_{i}^{(q)} \mathbf{I}\right)}{\left.\frac{d}{d s} \operatorname{det} \mathbf{F}^{(q)}(s)\right|_{s=\rho_{i}^{(q)}}} .
$$

The rest of the coefficients $\delta_{i, j}^{(q)}, j=2,3, \ldots$, can be found recursively by using the Taylor series of $\mathbf{F}^{(q)}(s)$ and $\mathbf{K}(s)$ around $\rho_{i}^{(q)}$ and equating all the coefficients of $\epsilon^{n}(n=2,3, \ldots)$ in $\operatorname{det} \mathbf{F}_{\epsilon}^{(q)}\left(\rho_{\epsilon, i}^{(q)}\right)=0$ to 0 .

\subsubsection{The perturbed left eigenvectors}

Using similar arguments as in [28, Theorem A.4], we can show that the rows of $\mathbf{L}_{\epsilon}^{(q)}$ can be defined with the aid of the adjoint matrix of $\mathbf{F}_{\epsilon}^{(q)}(s)$. In particular, if $\mathfrak{F}_{\epsilon}^{(q)}(s)$ is the adjoint of $\mathbf{F}_{\epsilon}^{(q)}(s)$, then the $i$ th row of $\mathbf{L}_{\epsilon}^{(q)}$, $i \in E$, is equal to any row of the matrix $\mathfrak{F}_{\epsilon}^{(q)}\left(\rho_{\epsilon, i}^{(q)}\right)$ that is not identically equal to zero. Thus, as a first step, we identify in the next theorem the exact form of a non-zero row of the matrix $\mathfrak{F}_{\epsilon}^{(q)}(s)$.

Theorem 4.3. There exists a row of the matrix $\mathfrak{F}_{\epsilon}^{(q)}(s)$ that is not identically equal to zero and takes the form

$$
\left(\operatorname{det}\left(\mathbf{T}^{(+)}+s \mathbf{I}\right):-\lambda^{(+)} \boldsymbol{\alpha}^{(+)} \operatorname{adj}\left(\mathbf{T}^{(+)}+s \mathbf{I}\right)\right) .
$$

If $q=0$ then $\operatorname{det} \mathbf{F}_{\epsilon}(0)=0$, which means that 0 is an eigenvalue of $\mathbf{F}_{\epsilon}(s)$. Therefore, for $q=s=0$, 
Equation (30) simplifies to

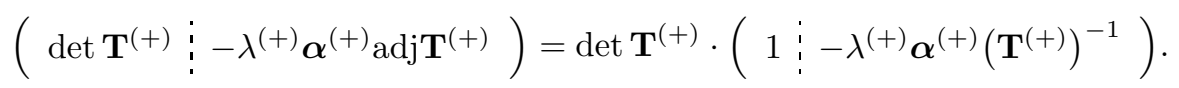

Proof. Recall that to find the $(i, j)$ element of the adjoint matrix $\mathfrak{F}^{(q)}(s)$, we calculate the determinant that occurs when we delete the $j$-row and the $i$-column of the initial matrix. Since the matrices $\mathbf{F}^{(q)}(s)$ and $\mathbf{F}_{\epsilon}^{(q)}(s)$ differ only on the $(1,1)$ element, the matrices $\mathfrak{F}^{(q)}(s)$ and $\mathfrak{F}_{\epsilon}^{(q)}(s)$ are equal on the 1st row and 1st column; i.e. if we delete the $(1,1)$ element either by deleting the 1st row or column, then the remaining minors are equal. Another key observation is that deleting the $(j, i)$ row-column combination in the matrix $\mathbf{F}_{\epsilon}^{(q)}(s)$, with $j, i \geq 2$, it means that we delete the $(j-1, i-1)$ row-column combination in the matrix $\mathbf{T}^{(+)}+s \mathbf{I}$. Therefore, it is easy to verify that

$$
\mathfrak{F}_{\epsilon}^{(q)}(s)=\mathfrak{F}^{(q)}(s)-\epsilon \mathfrak{K}(s)
$$

where

$$
\mathfrak{K}(s)=\left[\begin{array}{c|c}
0 & \mathbf{0} \\
\hline \mathbf{0} & s k(s) \operatorname{adj}\left(\mathbf{T}^{(+)}+s \mathbf{I}\right)
\end{array}\right] .
$$

Any row of $\mathfrak{F}_{\epsilon}^{(q)}(s)$ that is not identically equal to zero is a suitable candidate. Therefore, we can choose w.l.o.g. its 1st row because the matrix $\mathfrak{K}(s)$ has the 1 st row equal to zero and this simplifies the calculations. As a result, we need to find the 1 st row of $\mathfrak{F}^{(q)}(s)$. To do so, we use $(26)$ and the identity $\mathbf{F}_{\epsilon}^{(q)}(s) \mathfrak{F}_{\epsilon}^{(q)}(s)=\operatorname{det} \mathbf{F}_{\epsilon}^{(q)}(s) \mathbf{I}$. We obtain

$$
\begin{aligned}
\left(\mathbf{F}^{(q)}(s)-\epsilon \mathbf{K}(s)\right)\left(\mathfrak{F}^{(q)}(s)-\epsilon \mathfrak{K}(s)\right) & =\operatorname{det} \mathbf{F}^{(q)}(s) \mathbf{I}-\epsilon s k(s) \operatorname{det}\left(\mathbf{T}^{(+)}+s \mathbf{I}\right) \mathbf{I} \\
& \Rightarrow \\
\mathbf{F}^{(q)}(s) \mathfrak{K}(s)+\mathbf{K}(s) \mathfrak{F}^{(q)}(s) & =s k(s) \operatorname{det}\left(\mathbf{T}^{(+)}+s \mathbf{I}\right) \mathbf{I},
\end{aligned}
$$

since $\mathbf{K}(s) \mathfrak{K}(s)=\mathbf{0}$. The above equation means that only the diagonal elements of the matrix $\mathbf{F}^{(q)}(s) \mathfrak{K}(s)+$ $\mathbf{K}(s) \mathfrak{F}^{(q)}(s)$ are not identically equal to zero. Observe that we only need the 1st row of $\mathfrak{F}^{(q)}(s)$. Obviously, the $(1,1)$ element of $\mathfrak{F}^{(q)}(s)$ is equal to $\operatorname{det}\left(\mathbf{T}^{(+)}+s \mathbf{I}\right)$. To find the remaining elements, we find the 1 st row of $\mathbf{F}^{(q)}(s) \mathfrak{K}(s)$ as

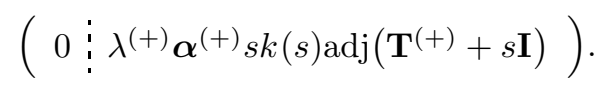

and then use the identity (34) to retrieve Equation (30).

As explained in the proof of Theorem 4.3, the vector (30) is also any row -not identically equal to zero- of $\mathfrak{F}^{(q)}(s)$. If $\mathbf{L}^{(q)}$ is the respective matrix of the phase-type base model, then its $i$ th row is equal to

$$
\left(\operatorname{det}\left(\mathbf{T}^{(+)}+\rho_{i}^{(q)} \mathbf{I}\right):-\lambda^{(+)} \boldsymbol{\alpha}^{(+)} \operatorname{adj}\left(\mathbf{T}^{(+)}+\rho_{i}^{(q)} \mathbf{I}\right)\right)
$$

The next theorem states that we can express $\mathbf{L}_{\epsilon}^{(q)}$ as a perturbation of $\mathbf{L}^{(q)}$. We denote by $\mathbf{A}_{i \bullet}$ and $\mathbf{A}_{\bullet} j$ the $i$ th row and $j$ th column of a matrix $\mathbf{A}$, and by $\mathbf{e}$ and $\mathbf{U}$ the row vector and matrix respectively with all elements equal to 1 .

Theorem 4.4. The matrix $\mathbf{L}_{\epsilon}^{(q)}$ admits a series expansion in $\epsilon$ with first term the matrix $\mathbf{L}^{(q)}$, i.e.

$$
\left(\mathbf{L}_{\epsilon}^{(q)}\right)_{i \bullet}=\left(\mathbf{L}^{(q)}\right)_{i \bullet}+\epsilon\left(\mathbf{H}_{1}^{(q)}\right)_{i \bullet}+O\left(\epsilon^{2} \mathbf{e}\right)
$$

where

$$
\left(\mathbf{H}_{1}^{(q)}\right)_{i \bullet}=\left.\delta_{i, 1}^{(q)} \frac{d}{d s}\left(\operatorname{det}\left(\mathbf{T}^{(+)}+s \mathbf{I}\right):-\lambda^{(+)} \boldsymbol{\alpha}^{(+)} \operatorname{adj}\left(\mathbf{T}^{(+)}+s \mathbf{I}\right)\right)\right|_{s=\rho_{i}^{(q)}}
$$


If $q=0$, then the first row of the matrix $\mathbf{H}_{1}^{(q)}$ is identically equal to zero.

Proof. Observe that the 1st element of the vector (30) is a polynomial in $s$ of degree $m^{(+)}$, while all its other elements are polynomials in $s$ of degree $m^{(+)}-1$. Therefore, the result is immediate by substituting in these polynomials $s=\rho_{i}^{(q)}+\epsilon \delta_{i, 1}^{(q)}+\epsilon^{2} \delta_{i, 2}^{(q)}+\ldots$ and finding the coefficient of $\epsilon$.

Deriving explicitly the series expansion of $\mathbf{L}_{\epsilon}^{(q)}$ is possible if one follows a similar recursive scheme to the one described in Remark 4.2. Moreover, we can find

$$
\left(\mathbf{L}_{\epsilon}^{(q)}\right)^{-1}=\left(\mathbf{L}^{(q)}\right)^{-1}-\epsilon\left(\mathbf{L}^{(q)}\right)^{-1} \mathbf{H}_{1}^{(q)}\left(\mathbf{L}^{(q)}\right)^{-1}+O\left(\epsilon^{2} \mathbf{U}\right)
$$

Finally, we calculate in the next section the matrix exponential $e^{-\mathbf{R}_{\epsilon}^{(q)} z}$ that appears in Equation (15).

\subsubsection{The perturbed matrix exponential}

Proposition 4.5. The series expansion of the matrix exponent $e^{-\mathbf{R}_{\epsilon}^{(q)} z}$ in Equation (15) takes the form

$$
\begin{aligned}
e^{-\mathbf{R}_{\epsilon}^{(q)} z}= & e^{-\mathbf{R}^{(q)} z}+\epsilon\left(\left(\mathbf{L}^{(q)}\right)^{-1} e^{-\mathbf{\Lambda}^{(q)} z} \mathbf{H}_{1}^{(q)}-z\left(\mathbf{L}^{(q)}\right)^{-1} e^{-\mathbf{\Lambda}^{(q)} z} \boldsymbol{\Delta}_{1}^{(q)} \mathbf{L}^{(q)}-\left(\mathbf{L}^{(q)}\right)^{-1} \mathbf{H}_{1}^{(q)} e^{-\mathbf{R}^{(q)} z}\right) \\
& +O\left(\epsilon^{2} \mathbf{U}\right) .
\end{aligned}
$$

Proof. Using the definition of the matrix exponent, i.e. $e^{\mathbf{A}}=\sum_{k=0}^{+\infty} \mathbf{A}^{k} / k$ ! for a square matrix $\mathbf{A}$, we can find that $e^{-\boldsymbol{\Lambda}_{e}^{(q)} z}=e^{-\boldsymbol{\Lambda}^{(q)} z}-\epsilon e^{-\boldsymbol{\Lambda}^{(q)} z} \boldsymbol{\Delta}_{1}^{(q)} z+O\left(\epsilon^{2} \mathbf{U}\right)$ because $\boldsymbol{\Lambda}^{(q)}$ and $\boldsymbol{\Delta}_{1}^{(q)}$ are diagonal matrices. Thus,

$$
\begin{aligned}
e^{-\mathbf{R}_{\epsilon}^{(q)} z}= & \left(\mathbf{L}_{\epsilon}^{(q)}\right)^{-1} e^{-\boldsymbol{\Lambda}_{\epsilon}^{(q)} z} \mathbf{L}_{\epsilon}^{(q)} \\
= & \left(\left(\mathbf{L}^{(q)}\right)^{-1}-\epsilon\left(\mathbf{L}^{(q)}\right)^{-1} \mathbf{H}_{1}^{(q)}\left(\mathbf{L}^{(q)}\right)^{-1}+O\left(\epsilon^{2} \mathbf{U}\right)\right) e^{-\left(\boldsymbol{\Lambda}^{(q)}+\epsilon \boldsymbol{\Delta}_{1}^{(q)}+O\left(\epsilon^{2} \mathbf{U}\right)\right) z} \\
& \times\left(\mathbf{L}^{(q)}+\epsilon \mathbf{H}_{1}^{(q)}+O\left(\epsilon^{2} \mathbf{U}\right)\right) \\
= & \left(\left(\mathbf{L}^{(q)}\right)^{-1}-\epsilon\left(\mathbf{L}^{(q)}\right)^{-1} \mathbf{H}_{1}^{(q)}\left(\mathbf{L}^{(q)}\right)^{-1}+O\left(\epsilon^{2} \mathbf{U}\right)\right)\left(e^{-\boldsymbol{\Lambda}^{(q)} z}-\epsilon e^{-\boldsymbol{\Lambda}^{(q)} z} \boldsymbol{\Delta}_{1}^{(q)} z+O\left(\epsilon^{2} \mathbf{U}\right)\right) \\
& \times\left(\mathbf{L}^{(q)}+\epsilon \mathbf{H}_{1}^{(q)}+O\left(\epsilon^{2} \mathbf{U}\right)\right) \\
= & e^{-\mathbf{R}^{(q)} z}+\epsilon\left(\left(\mathbf{L}^{(q)}\right)^{-1} e^{-\mathbf{\Lambda}^{(q)} z} \mathbf{H}_{1}^{(q)}-z\left(\mathbf{L}^{(q)}\right)^{-1} e^{-\boldsymbol{\Lambda}^{(q)} z} \mathbf{\Delta}_{1}^{(q)} \mathbf{L}^{(q)}-\left(\mathbf{L}^{(q)}\right)^{-1} \mathbf{H}_{1}^{(q)} e^{-\mathbf{R}^{(q)} z}\right)+O\left(\epsilon^{2} \mathbf{U}\right) .
\end{aligned}
$$

Corollary 4.6. The series expansion of the q-resolvent measure $\left(\mathbf{r}_{\epsilon}^{(q)}(u, z)\right)_{(1,1)}$ in Lemma 3.3 takes the form

$$
\left(\mathbf{r}_{\epsilon}^{(q)}(u, z)\right)_{(1,1)}=\left(\mathbf{r}^{(q)}(u, z)\right)_{(1,1)}+\epsilon v^{(q)}(u, z)+O\left(\epsilon^{2}\right)
$$

for all $u, z \geq 0$, where

$$
\begin{aligned}
v^{(q)}(u, z)= & \lambda^{(-)}\left(\mu_{h} F_{h}^{e}(u)-\mu_{p} F_{p}^{e}(u)\right) *\left(\mathbf{W}^{(q)}(d u)\right)_{(1,1)} *\left(\mathbf{W}^{(q)}(d u)\right)_{1 \bullet} \cdot\left(e^{-\mathbf{R}^{(q)} z}\right)_{\bullet 1} \\
& +\left(\mathbf{W}^{(q)}(u)\right)_{1 \bullet} \cdot\left(\left(\mathbf{L}^{(q)}\right)^{-1} e^{-\mathbf{\Lambda}^{(q)} z} \mathbf{H}_{1}^{(q)}-z\left(\mathbf{L}^{(q)}\right)^{-1} e^{-\mathbf{\Lambda}^{(q)} z} \boldsymbol{\Delta}_{1}^{(q)} \mathbf{L}^{(q)}-\left(\mathbf{L}^{(q)}\right)^{-1} \mathbf{H}_{1}^{(q)} e^{-\mathbf{R}^{(q)} z}\right)_{\bullet 1} \\
& -\lambda^{(-)} \int_{0}^{u-z}\left(\mu_{h} F_{h}^{e}(u-z-x)-\mu_{p} F_{p}^{e}(u-z-x)\right)\left(\mathbf{W}^{(q)}(d x)\right)_{(1,1)}^{* 2} .
\end{aligned}
$$

Proof. From Equations (23) and (25), we find that the 1st row of the matrix $\mathbf{W}_{\epsilon}^{(q)}(x)$ is given by

$$
\left(\mathbf{W}_{\epsilon}^{(q)}(x)\right)_{1 \bullet}=\left(\mathbf{W}^{(q)}(x)\right)_{1 \bullet}+\epsilon \lambda^{(-)}\left(\mu_{h} F_{h}^{e}(x)-\mu_{p} F_{p}^{e}(x)\right) *\left(\mathbf{W}^{(q)}(d x)\right)_{(1,1)} *\left(\mathbf{W}^{(q)}(d x)\right)_{1}+O\left(\epsilon^{2} \mathbf{U}\right) .
$$


Thus, to retrieve Equation (38), we need to combine the above formula with Lemma 3.3 and Proposition 4.5, and identify the coefficient of $\epsilon$.

\section{Corrected approximation for the Gerber-Shiu function}

Combining the previous results produces the following expansion for the Gerber-Shiu function.

Theorem 5.1. Let $q>0$. The GS function $G S_{\omega}^{\epsilon}(u, q)$ in Equation (15) has a series expansion as follows

$$
\begin{aligned}
G S_{\omega}^{\epsilon}(u, q)= & G S_{\omega}(u, q) \\
& +\epsilon A(q) \lambda^{(-)}\left(\int_{[0, \infty)} \int_{[0, \infty)} \omega(y, z) \mathbf{r}^{(q)}(u, z)_{(1,1)}\left(F_{h}(z+d y)-F_{p}(z+d y)\right) d z\right. \\
& \left.+\int_{[0, \infty)} \int_{[0, \infty)} \omega(y, z) v^{(q)}(u, z) F_{p}(z+d y) d z\right)+O\left(\epsilon^{2}\right)
\end{aligned}
$$

where $G S_{\omega}(u, q)$ and $\mathbf{r}^{(q)}(u, z)_{(1,1)}$ are evaluated respectively through Equations (9) and (14) with $\epsilon=0$.

Note that $G_{\omega}(u, q)$ is the GS function for the base model without the heavy-tailed component $F_{h}$ and having only phase-type upward and downwards jumps. Moreover, the error that this approximation makes is of order $O(\epsilon)$. By keeping only the two first terms of the series expansion of $G S_{\omega}^{\epsilon}(u, q)$ in Theorem 5.1, we can define an approximation for the GS function that makes an error of order $O\left(\epsilon^{2}\right)$. We call it corrected phasetype approximation because the $\epsilon$-order term corrects the behavior of $G S_{\omega}(u, q)$, which is by itself a phase-type approximation for $G S_{\omega}^{\epsilon}(u, q)$.

Approximation 5.2. For $q>0$, the corrected phase-type approximation for $G S_{\omega}^{\epsilon}(u, q)$ is defined as

$$
\begin{aligned}
\widehat{G S_{\omega}^{\epsilon}}(u, q):= & G S_{\omega}(u, q) \\
& +\epsilon A(q) \lambda^{(-)}\left(\int_{[0, \infty)} \int_{[0, \infty)} \omega(y, z) \mathbf{r}^{(q)}(u, z)_{(1,1)}\left(F_{h}(z+d y)-F_{p}(z+d y)\right) d z\right. \\
& \left.+\int_{[0, \infty)} \int_{[0, \infty)} \omega(y, z) v^{(q)}(u, z) F_{p}(z+d y) d z\right),
\end{aligned}
$$

where $G S_{\omega}(u, q)=\lambda^{(-)} \int_{[0, \infty)} \int_{[0, \infty)} \omega(y, z) \mathbf{r}^{(q)}(u, z)_{(1,1)} F_{p}(z+d y) d z$

Observe that one can construct even more accurate approximations for $G S_{\omega}^{\epsilon}(u, q)$ by simply keeping more terms in the series expansion of Theorem 5.1. More precisely, an approximation including up to the $\epsilon^{n}$-order term gives an error of $O\left(\epsilon^{n+1}\right)$. However, calculating higher order terms is not always easy. The difficulty mainly comes from the evaluation of the series expansion of the matrix $e^{-\mathbf{R}_{\epsilon}^{(q)} z}$ because the scale matrix $\mathbf{W}_{\epsilon}^{(q)}(u)$ has an explicit series expansion. In certain models where the series expansion of $e^{-\mathbf{R}_{\epsilon}^{(q)} z}$ can be calculated explicitly, then it is also possible to derive the whole series expansion for $G S_{\omega}^{\epsilon}(u, q)$ as well. One such particular example is the ruin probability in the classical Cramér-Lundberg.

\subsection{Application - Ruin probability in the Cramér-Lundberg risk model}

In this section, we derive the corrected phase-type approximation for the ruin probability of the classical Cramér-Lundberg risk model. Since there are no positive jumps, $\lambda^{(+)}=m^{(+)}=0, Y_{\epsilon}(t) \equiv X_{\epsilon}(t)$, and $A(q)=1$. Moreover, we must take $\omega \equiv 1$ and $q=0$. In fact, to apply Approximation 5.2, we should take $q \downarrow 0$ and treat both sides of this approximation in the limiting sense. Note that this procedure is correct since both sides of this identity converge as $q \downarrow 0$. According to Section 3, the matrix $\mathbf{F}_{\epsilon}(s)$, which is actually equal to the scalar 
$\phi_{\epsilon}(s)$ in Equation (12), has zero as its only single eigenvalue and thus $e^{-\mathbf{R}_{\epsilon}^{(q)}} z=1$, which makes the middle term in (38) vanish. Finally, the positive safety loading condition takes the form $\lambda^{(-)}(1-\epsilon) \mu_{p}+\lambda^{(-)} \epsilon \mu_{h}<c$ for the mixture model and $\lambda^{(-)} \mu_{p} / c<1$ for the base model.

For the base model, we calculate the scale matrix/function through

$$
(\mathbf{F}(s))^{-1}=\frac{1}{c s} \cdot \frac{1}{1-\frac{\lambda^{(-)} \mu_{p}}{c} \tilde{F}_{p}^{e}(s)}=\frac{1}{c s} \sum_{n=0}^{+\infty}\left(\frac{\lambda^{(-)} \mu_{p}}{c}\right)^{n}\left(\tilde{F}_{p}^{e}(s)\right)^{n} \Rightarrow \mathbf{W}(x)=\frac{1}{c} \sum_{n=0}^{+\infty}\left(\frac{\lambda^{(-)} \mu_{p}}{c}\right)^{n}\left(F_{p}^{e}\right)^{* n}(x)
$$

while the resolvent is equal to

$$
\mathbf{r}(u, z)=\mathbf{W}(u)-\mathbf{W}(u-z)=\frac{1}{c} \sum_{n=0}^{+\infty}\left(\frac{\lambda^{(-)} \mu_{p}}{c}\right)^{n}\left(\left(F_{p}^{e}\right)^{* n}(u)-\left(F_{p}^{e}\right)^{* n}(u-z)\right)
$$

If $M=-\inf _{0 \leq t<\infty}(X(t)-X(0))$ and $\psi(u)$ is the ruin probability of the base model, then the PollaczeckKhinchine formula gives

$$
\begin{gathered}
\mathbb{P}(M \leq u)=1-\psi(u)=\left(1-\frac{\lambda^{(-)} \mu_{p}}{c}\right) \sum_{n=0}^{+\infty}\left(\frac{\lambda^{(-)} \mu_{p}}{c}\right)^{n}\left(F_{p}^{e}\right)^{* n}(u)=\left(c-\lambda^{(-)} \mu_{p}\right) \mathbf{W}(u) \\
\Rightarrow \quad \\
\mathbf{r}(u, z)=\frac{1}{c-\lambda^{(-)} \mu_{p}}(\mathbb{P}(M \leq u)-\mathbb{P}(M \leq u-z)) .
\end{gathered}
$$

In addition, we calculate

$$
\begin{aligned}
v^{(q)}(u, z)= & v^{(0)}(u, z)=\lambda^{(-)}\left(\mu_{h} F_{h}^{e}(u)-\mu_{p} F_{p}^{e}(u)\right) *(\mathbf{W}(d u))^{* 2} \\
& -\lambda^{(-)} \int_{0}^{u-z}\left(\mu_{h} F_{h}^{e}(u-z-x)-\mu_{p} F_{p}^{e}(u-z-x)\right)(\mathbf{W}(d x))^{* 2} \\
= & \frac{\lambda^{(-)}}{\left(c-\lambda^{(-)} \mu_{p}\right)^{2}}\left(\mu_{h} F_{h}^{e}(u)-\mu_{p} F_{p}^{e}(u)\right) *(\mathbb{P}(M \in d u))^{* 2} \\
& -\frac{\lambda^{(-)}}{\left(c-\lambda^{(-)} \mu_{p}\right)^{2}} \int_{0}^{u-z}\left(\mu_{h} F_{h}^{e}(u-z-x)-\mu_{p} F_{p}^{e}(u-z-x)\right)(\mathbb{P}(M \in d x))^{* 2} .
\end{aligned}
$$

Noting that $\int_{0}^{\infty} F_{h}(z+d y)=\mu_{h} F_{h}^{e}(d z) / d z$ and $\int_{0}^{\infty} F_{p}(z+d y)=\mu_{p} F_{p}^{e}(d z) / d z$ and letting $M^{*} \stackrel{\mathfrak{D}}{=} M$, $B^{e *} \stackrel{\mathfrak{D}}{=} B^{e}$, and $C^{e *} \stackrel{\mathfrak{D}}{=} C^{e}$, where all are mutually independent, the approximation for the ruin probability $\widehat{\psi}_{\epsilon}(u)$ in Approximation 5.2 then takes the form

$$
\begin{aligned}
\widehat{\psi}_{\epsilon}(u)= & \widehat{G S_{1}^{\epsilon}}(u, 0)=\psi(u)+\epsilon \lambda^{(-)}\left(\int_{[0, \infty)} \mathbf{r}(u, z)\left(\mu_{h} F_{h}^{e}(d z)-\mu_{p} F_{p}^{e}(d z)\right)+\int_{[0, \infty)} v^{(q)}(u, z) \mu_{p} F_{p}^{e}(d z)\right) \\
= & \psi(u)+\frac{\epsilon \lambda^{(-)}\left(\mu_{h}-\mu_{p}\right)}{c-\lambda^{(-)} \mu_{p}} \mathbb{P}(M \leq u)-\frac{\epsilon \lambda^{(-)}}{c-\lambda^{(-)} \mu_{p}}\left(\mu_{h} \mathbb{P}\left(M+C^{e} \leq u\right)-\mu_{p} \mathbb{P}\left(M+B^{e} \leq u\right)\right) \\
& +\frac{\epsilon \lambda^{(-)} \mu_{p}}{\left(c-\lambda^{(-)} \mu_{p}\right)^{2}}\left(\lambda^{(-)} \mu_{h} \mathbb{P}\left(M+M^{*}+C^{e} \leq u\right)-\lambda^{(-)} \mu_{p} \mathbb{P}\left(M+M^{*}+B^{e} \leq u\right)\right) \\
& -\frac{\epsilon \lambda^{(-)} \mu_{p}}{\left(c-\lambda^{(-)} \mu_{p}\right)^{2}}\left(\lambda^{(-)} \mu_{h} \mathbb{P}\left(M+M^{*}+C^{e}+B^{e} \leq u\right)-\lambda^{(-)} \mu_{p} \mathbb{P}\left(M+M^{*}+B^{e}+B^{e *} \leq u\right)\right)=\ldots \\
= & \psi(u)+\frac{\epsilon \lambda^{(-)} \mu_{h}}{c-\lambda^{(-)} \mu_{p}}\left(\mathbb{P}\left(M+M^{*}+C^{e}>u\right)-\mathbb{P}(M>u)\right) \\
& -\frac{\epsilon \lambda^{(-)} \mu_{p}}{c-\lambda^{(-)} \mu_{p}}\left(\left(\mathbb{P}\left(M+M^{*}+B^{e}>u\right)-\mathbb{P}(M>u)\right) .\right.
\end{aligned}
$$


Notice that we have used the identity $\mathbb{P}(M \in d x) * F_{p}^{e}(d x)=\mathbb{P}\left(M+B^{e} \in d x\right)=\frac{c}{\lambda^{(-)} \mu_{p}} \mathbb{P}(M \in d x)-\frac{c-\lambda^{(-)} \mu_{p}}{\lambda^{(-)} \mu_{p}}$ to simplify the convoluted probabilities $\mathbb{P}\left(M+M^{*}+C^{e}+B^{e} \leq u\right)$ and $\mathbb{P}\left(M+M^{*}+B^{e}+B^{e *} \leq u\right)$, while $\int_{0}^{\infty} F_{h}^{e}(d z)=\int_{0}^{\infty} F_{p}^{e}(d z)=1$, because $F_{h}^{e}$ and $F_{p}^{e}$ are distributions. The above result is in accord with [29], which further accommodates the whole series expansion of $\widehat{\psi}_{\epsilon}(u)$.

\subsection{Heavy-tailed asymptotics}

Some of the summands in the $\epsilon$ term of our corrected phase-type approximation $\widehat{G S_{\omega}^{\epsilon}}(u, q)$ are convolutions of a single excess heavy-tailed claim size $C^{e}$ with some light tailed distributions. We make rigorous in this section that these summands administer a heavy-tailed behavior to the approximation. Note that we don't aim at deriving exact tail asymptotics. We rather intend to provide a feeling for the asymptotic behavior of the approximation and thus treat this section more as a discussion than a strict proof.

For our purposes, we assume that $F_{h}$ belongs to the class of long-tailed distributions, that is, for any fixed $y>0$ we have $\lim _{x \rightarrow \infty} \bar{F}_{h}(x+y) / \bar{F}_{h}(x)=1$. In addition, recall that $\omega$ is a bounded function, i.e. $\omega \leq a$ for some constant $a>0$. Finally, we only focus on the case $q>0$, since the proof for $q=0$ can be handled as a limiting case.

Since $\omega$ is bounded, it is clear from Equation (2) that the GS function is bounded from above by the ruin probability multiplied by a constant. In this case, from [3, Lemma 1 \& Equation (19)],

$$
G S_{\omega}(u, q)=A(q) \lambda^{(-)} \int_{[0, \infty)} \int_{[0, \infty)} \omega(y, z) \mathbf{r}^{(q)}(u, z)_{(1,1)} F_{p}(z+d y) d z \leq \beta e^{-\gamma u}
$$

for some constant $\beta$ and a strictly positive number $\gamma$. We will show that each of the remaining two terms of Approximation 5.2 can be split in a part which is also bounded by an exponential and a heavy-tailed part that is responsible for the tail behavior of the approximation $\widehat{G S_{\omega}^{\epsilon}}(u, q)$.

Term 1. We start our discussion with the term

$$
\epsilon A(q) \lambda^{(-)} \int_{[0, \infty)} \int_{[0, \infty)} \omega(y, z) v^{(q)}(u, z) F_{p}(z+d y) d z
$$

where $v^{(q)}(u, z)$ is provided in Corollary 4.6. Clearly, we can write $v^{(q)}(u, z)=v_{p}^{(q)}(u, z)+v_{h}^{(q)}(u, z)$, where

$$
\begin{aligned}
v_{p}^{(q)}(u, z)= & -\lambda^{(-)} \mu_{p} F_{p}^{e}(u) *\left(\mathbf{W}^{(q)}(d u)\right)_{(1,1)} *\left(\mathbf{W}^{(q)}(d u)\right)_{1 \bullet} \cdot\left(e^{-\mathbf{R}^{(q)} z}\right)_{\bullet 1} \\
& +\left(\mathbf{W}^{(q)}(u)\right)_{1 \bullet} \cdot\left(\left(\mathbf{L}^{(q)}\right)^{-1} e^{-\mathbf{\Lambda}^{(q)} z} \mathbf{H}_{1}^{(q)}-z\left(\mathbf{L}^{(q)}\right)^{-1} e^{-\mathbf{\Lambda}^{(q)} z} \boldsymbol{\Delta}_{1}^{(q)} \mathbf{L}^{(q)}-\left(\mathbf{L}^{(q)}\right)^{-1} \mathbf{H}_{1}^{(q)} e^{-\mathbf{R}^{(q)} z}\right)_{\bullet 1} \\
& +\lambda^{(-)} \int_{0}^{u-z} \mu_{p} F_{p}^{e}(u-z-x)\left(\mathbf{W}^{(q)}(d x)\right)_{(1,1)}^{* 2}
\end{aligned}
$$

and

$$
\begin{aligned}
v_{h}^{(q)}(u, z)= & \lambda^{(-)} \mu_{h} F_{h}^{e}(u) *\left(\mathbf{W}^{(q)}(d u)\right)_{(1,1)} *\left(\mathbf{W}^{(q)}(d u)\right)_{1 \bullet} \cdot\left(e^{-\mathbf{R}^{(q)} z}\right)_{\bullet 1} \\
& -\lambda^{(-)} \int_{0}^{u-z} \mu_{h} F_{h}^{e}(u-z-x)\left(\mathbf{W}^{(q)}(d x)\right)_{(1,1)}^{* 2} .
\end{aligned}
$$

Lemma 5.3. The term

$$
\epsilon A(q) \lambda^{(-)} \int_{[0, \infty)} \int_{[0, \infty)} \omega(y, z) v_{p}^{(q)}(u, z) F_{p}(z+d y) d z
$$

is bounded by an exponential. 
Proof. Indeed, from [9, Equation (2.3)], [18, Theorem 1], and [17, Corollary 1], we have that

$$
\lim _{u \rightarrow+\infty} e^{-\mathbf{\Lambda}^{(q)} u} \mathbf{W}^{(q)}(u)=\lim _{u \rightarrow+\infty} \mathbf{W}^{(q)}(u) e^{-\mathbf{R}^{(q)} u}=\mathbf{L}^{(q)}
$$

Now using the fact that $\omega$ is bounded, we can write

$$
\begin{aligned}
\epsilon A(q) \lambda^{(-)} & \int_{0}^{\infty} \int_{0}^{\infty} \omega(y, z) v_{p}^{(q)}(u, z) F_{p}(z+d y) d z \leq \epsilon A(q) \lambda^{(-)} a \int_{0}^{\infty} v_{p}^{(q)}(u, z) \bar{F}_{p}(z) d z \\
& =\epsilon A(q) \lambda^{(-)} a \int_{0}^{u} v_{p}^{(q)}(u, z) \bar{F}_{p}(z) d z+\epsilon A(q) \lambda^{(-)} a \int_{u}^{\infty} v_{p}^{(q)}(u, z) \bar{F}_{p}(z) d z \\
& \leq \epsilon A(q) \lambda^{(-)} a \underbrace{\int_{0}^{u} v_{p}^{(q)}(u, z) \bar{F}_{p}(z) d z}_{:=I_{1}}+\epsilon A(q) \lambda^{(-)} a \underbrace{\bar{F}_{p}(u) \int_{u}^{\infty} v_{p}^{(q)}(u, z) d z}_{:=I_{2}} .
\end{aligned}
$$

It is well known that any phase-type distribution is light-tailed, that is there exist $\gamma_{p}$ and $\beta_{p}$ such that

$$
\lim _{u \rightarrow+\infty} \bar{F}_{p}(u) e^{\gamma_{p} u}=\beta_{p}>0
$$

Note that the integral $\int_{u}^{\infty} e^{-\mathbf{R}^{(q)} z} d z$ is of order $e^{-\mathbf{R}^{(q)} u}$. Consequently, it follows that $\int_{u}^{\infty} v_{p}^{(q)}(u, z) d z$ tends to a constant as $u \rightarrow+\infty$ and hence the term $I_{2}$ is dominated by some exponential function due to (42).

For $I_{1}$, observe that each summand of $v_{p}^{(q)}(u, z)$ behaves like $\mathbf{W}^{(q)}(u)$ for fixed $z \leq u$. In other words, there exists by (41) a $\gamma_{W}>0$ such that $\lim _{u \rightarrow+\infty} \mathbf{W}^{(q)}(u) e^{\gamma_{W} u}=\mathbf{L}_{0}^{(q)}$ for a non-zero and finite matrix $\mathbf{L}_{0}^{(q)}$. As a result, all the increments in (39) multiplied by $e^{\gamma_{W} u}$ tend to some constants, the sum of which must be equal to zero because the Gerber-Shiu function that dominates the sum goes to zero. The asymptotics of $I_{1}$ are then determined by the sum of the speeds that each increment tends to a constant. Remark that the speeds of convergence of $-\lambda^{(-)} \mu_{p} \int_{0}^{u} F_{p}^{e}(u) *\left(\mathbf{W}^{(q)}(d u)\right)_{(1,1)} *\left(\mathbf{W}^{(q)}(d u)\right)_{1} \cdot\left(e^{-\mathbf{R}^{(q)} z}\right)_{\bullet 1} d z$ and $\lambda^{(-)} \int_{0}^{u} \int_{0}^{u-z} \mu_{p} F_{p}^{e}(u-$ $z-x)\left(\mathbf{W}^{(q)}(d x)\right)_{(1,1)}^{* 2} d z$ are of order $\bar{F}_{p}(u)$ and therefore exponential by (42).

To analyze the second increment in (39) and complete the proof, it suffices to prove that the speed of convergence in (41) is exponential. We focus on the first convergence, while the second can be analyzed in a similar way. We have from [18, Theorem 1] that $e^{-\Lambda^{(q)} u} \mathbf{W}^{(q)}(u)=\mathbf{L}^{(q)}(u)$, where $\mathbf{L}^{(q)}(u)$ is the matrix of expected occupation times at 0 up to the first passage over $u$ of the process $Y(t)$ exponentially killed with intensity $q>0$, i.e. up to $\tau_{u}^{+}=\inf \{t \geq 0: Y(t) \geq u\}$, where $Y(t)$ starts right now at level 0 . From the comment below this aforementioned theorem, it follows that $\lim _{u \rightarrow \infty} \mathbf{L}^{(q)}(u)=\mathbf{L}^{(q)}$, where $\mathbf{L}^{(q)}$ is the matrix of expected occupation times at 0 . If $\tau_{u}=\inf \{t \geq 0: Y(t)=-u\}$ is the first hitting time of level $-u$, it then follows from $[18$, Equation (10)] that

$$
\mathbf{L}^{(q)}-\mathbf{L}^{(q)}(u)=\mathbb{P}_{0}\left(J\left(\tau_{u}^{+}\right)\right) \mathbb{P}_{0}\left(J\left(\tau_{u}\right), \tau<+\infty\right) \mathbf{L}^{(q)}
$$

To prove that the speed is exponential, it suffices then to show that $\mathbb{P}_{0}\left(J\left(\tau_{u}\right)\right)$ has an exponential bound. Consequently, it holds that $\mathbb{P}\left(J\left(\tau_{u}\right)\right)=\int_{0}^{\infty} \mathbb{P}_{u}(|Y(\tau)| \in d y, J(\tau), \tau<+\infty) \mathbb{P}_{0}\left(J\left(\tau_{y}^{+}\right)\right) d y$ from [18, Proposition 7] and the Markov property. Thus, it only remains to show that $\psi(u)=\mathbb{P}_{u}(J(\tau), \tau<+\infty)$ has an exponential bound, which is true due to [3, Equation (19)].

For the term

$$
\epsilon A(q) \lambda^{(-)} \int_{[0, \infty)} \int_{[0, \infty)} \omega(y, z) v_{h}^{(q)}(u, z) F_{p}(z+d y) d z
$$

we first analyze further $v_{h}^{(q)}(u, z)$ as follows

$$
v_{h}^{(q)}(u, z)=\lambda^{(-)} \mu_{h} \int_{0}^{u} F_{h}^{e}(u-x)\left(\mathbf{W}^{(q)}(d x)\right)_{(1,1)} *\left(\mathbf{W}^{(q)}(d x)\right)_{1 \bullet} \cdot\left(e^{-\mathbf{R}^{(q)} z}\right)_{\bullet 1}
$$




$$
\begin{aligned}
& -\lambda^{(-)} \int_{0}^{u-z} \mu_{h} F_{h}^{e}(u-z-x)\left(\mathbf{W}^{(q)}(d x)\right)_{(1,1)}^{* 2} \\
= & \lambda^{(-)} \mu_{h} \int_{0}^{u}\left(\mathbf{W}^{(q)}(d x)\right)_{(1,1)} *\left(\mathbf{W}^{(q)}(d x)\right)_{1} \bullet\left(e^{-\mathbf{R}^{(q)} z}\right)_{\bullet 1}-\lambda^{(-)} \mu_{h}\left(\mathbf{W}^{(q)}(u-z)\right)_{(1,1)}^{*(2)} \\
& -\lambda^{(-)} \mu_{h} \int_{0}^{u} \bar{F}_{h}^{e}(u-x)\left(\mathbf{W}^{(q)}(d x)\right)_{(1,1)} *\left(\mathbf{W}^{(q)}(d x)\right)_{1} \bullet\left(e^{-\mathbf{R}^{(q)} z}\right)_{\bullet 1} \\
& +\lambda^{(-)} \mu_{h} \int_{0}^{u-z} \bar{F}_{h}^{e}(u-z-x)\left(\mathbf{W}^{(q)}(d x)\right)_{(1,1)}^{* 2},
\end{aligned}
$$

where we used the notation $\left(\mathbf{W}^{(q)}(x)\right)_{(1,1)}^{*(2)}=\left(\mathbf{W}^{(q)}(d x)\right)_{(1,1)} *\left(\mathbf{W}^{(q)}(x)\right)_{(1,1)}$. Thus,

$$
\begin{aligned}
\epsilon A(q) \lambda^{(-)} \int_{0}^{\infty} \int_{0}^{\infty} \omega(y, z) v_{h}^{(q)}(u, z) F_{p}(z+d y) d z \\
\leq \epsilon a A(q) \lambda^{(-)} \mu_{p} \lambda^{(-)} \mu_{h} \int_{0}^{\infty} \int_{0}^{u}\left(\mathbf{W}^{(q)}(d x)\right)_{(1,1)} *\left(\mathbf{W}^{(q)}(d x)\right)_{1} \cdot\left(e^{-\mathbf{R}^{(q)} z}\right)_{\bullet} F_{p}^{e}(d z) \\
\quad+\epsilon a A(q) \lambda^{(-)} \mu_{p} \lambda^{(-)} \mu_{h} \int_{0}^{u}\left(\mathbf{W}^{(q)}(u-z)\right)_{(1,1)}^{*(2)} F_{p}^{e}(d z) \\
\quad-\epsilon A(q) \lambda^{(-)} \int_{0}^{\infty} \int_{0}^{\infty} \omega(y, z) \lambda^{(-)} \mu_{h} \int_{0}^{u} \bar{F}_{h}^{e}(u-x)\left(\mathbf{W}^{(q)}(d x)\right)_{(1,1)} *\left(\mathbf{W}^{(q)}(d x)\right)_{1} \cdot\left(e^{-\mathbf{R}^{(q)} z}\right)_{\bullet 1} F_{p}(z+d y) d z \\
\quad+\epsilon A(q) \lambda^{(-)} \int_{0}^{\infty} \int_{0}^{\infty} \omega(y, z)\left(\lambda^{(-)} \mu_{h} \int_{0}^{u-z} \bar{F}_{h}^{e}(u-z-x)\left(\mathbf{W}^{(q)}(d x)\right)_{(1,1)}^{* 2}\right) F_{p}(z+d y) d z \\
=\epsilon a A(q) \lambda^{(-)} \mu_{p} \lambda^{(-)} \mu_{h}\left(\mathbf{W}^{(q)}(d u)\right)_{(1,1)} *\left(\mathbf{W}^{(q)}(u)\right)_{1} \cdot\left(\left(\mathbf{L}^{(q)}\right)^{-1} \operatorname{diag}\left(e^{\tilde{F}_{p}^{e}\left(\rho_{i}^{(q)}\right)}\right)_{i \in E} \mathbf{L}^{(q)}\right)_{\bullet 1} \\
\quad+\epsilon a A(q) \lambda^{(-)} \mu_{p} \lambda^{(-)} \mu_{h} F_{p}^{e}(u) *\left(\mathbf{W}^{(q)}(d u)\right)_{(1,1)}^{* 2}+\epsilon A(q) \lambda^{(-)} \int_{0}^{u} \bar{F}_{h}^{e}(u-x) \varphi(x) d x,
\end{aligned}
$$

with

$$
\begin{aligned}
\varphi(x)= & -\lambda^{(-)} \mu_{h}\left(\mathbf{W}^{(q)}(d x)\right)_{(1,1)} *\left(\mathbf{W}^{(q)}(d x)\right)_{1} \bullet \int_{0}^{\infty} \int_{0}^{\infty} \omega(y, z)\left(e^{-\mathbf{R}^{(q)} z}\right)_{\bullet 1} F_{p}(z+d y) d z \\
& +\lambda^{(-)} \mu_{h}\left(\mathbf{W}^{(q)}(d x)\right)_{(1,1)}^{* 2} \int_{0}^{\infty} \int_{0}^{x} \omega(y, z) F_{p}(z+d y) d z .
\end{aligned}
$$

Using similar arguments as in Lemma 5.3, we can prove that the first two terms in (43) are bounded by an exponential.

Term 2. We continue now our analysis with the term

$$
\epsilon A(q) \lambda^{(-)} \int_{[0, \infty)} \int_{[0, \infty)} \omega(y, z) \mathbf{r}^{(q)}(u, z)_{(1,1)} F_{h}(z+d y) d z .
$$

Using $e^{-\mathbf{R}^{(q)} z}=\left(\mathbf{L}^{(q)}\right)^{-1} e^{-\mathbf{\Lambda}^{(q)} z} \mathbf{L}^{(q)}$, we then write

$$
\begin{aligned}
\epsilon A(q) \lambda^{(-)} & \int_{0}^{\infty} \int_{0}^{\infty} \omega(y, z) \mathbf{r}^{(q)}(u, z)_{(1,1)} F_{h}(z+d y) d z \leq \epsilon A(q) \lambda^{(-)} a \mu_{h} \int_{0}^{\infty} \mathbf{r}^{(q)}(u, z)_{(1,1)} F_{h}^{e}(d z) \\
= & \epsilon A(q) \lambda^{(-)} a \mu_{h}\left(\mathbf{W}^{(q)}(u)\right)_{1} \cdot \int_{0}^{\infty}\left(e^{-\mathbf{R}^{(q)} z}\right)_{\bullet 1} F_{h}^{e}(d z)-\epsilon A(q) \lambda^{(-)} a \mu_{h} \int_{0}^{u}\left(\mathbf{W}^{(q)}(u-z)\right)_{(1,1)} F_{h}^{e}(d z) \\
= & \epsilon A(q) \lambda^{(-)} a \mu_{h}\left(\mathbf{W}^{(q)}(u)\right)_{1 \bullet} \cdot\left(\left(\mathbf{L}^{(q)}\right)^{-1} \operatorname{diag}\left(e^{\tilde{F}_{h}^{e}\left(\rho_{i}^{(q)}\right)}\right)_{i \in E} \mathbf{L}^{(q)}\right)_{\bullet 1} \\
& -\left.\epsilon A(q) \lambda^{(-)} a \mu_{h} \bar{F}_{h}^{e}(u-z)\left(\mathbf{W}^{(q)}(z)\right)_{(1,1)}\right|_{0} ^{u}+\epsilon A(q) \lambda^{(-)} a \mu_{h} \int_{0}^{u} \bar{F}_{h}^{e}(u-z)\left(\mathbf{W}^{(q)}(d z)\right)_{(1,1)} \\
= & \epsilon A(q) \lambda^{(-)} a \mu_{h}\left(\mathbf{W}^{(q)}(u)\right)_{1} \cdot\left(\left(\mathbf{L}^{(q)}\right)^{-1} \operatorname{diag}\left(e^{\tilde{F}_{h}^{e}\left(\rho_{i}^{(q)}\right)}\right)_{i \in E} \mathbf{L}^{(q)}\right)_{\bullet 1}-\epsilon A(q) \lambda^{(-)} a \mu_{h}\left(\mathbf{W}^{(q)}(u)\right)_{(1,1)}
\end{aligned}
$$




$$
+\epsilon A(q) \lambda^{(-)} a \mu_{h}\left(\mathbf{W}^{(q)}(0)\right)_{(1,1)} \bar{F}_{h}^{e}(u)+\epsilon A(q) \lambda^{(-)} a \mu_{h} \int_{0}^{u} \bar{F}_{h}^{e}(u-z)\left(\mathbf{W}^{(q)}(d z)\right)_{(1,1)} .
$$

Again, we can prove that the first two terms in (44) are bounded by an exponential by using similar arguments as in Lemma 5.3.

To show now the main result of this section, we need additional lemma. We need also the definition of $h$ insensitivity from [12], which we call here $\phi$-insensitivity to avoid confusion with the index $h$ in $F_{h}^{e}$. In particular, given a non-decreasing function $\phi$ on $\mathbb{R}^{+}$such that $\phi(x) \rightarrow \infty$ as $x \rightarrow \infty$, a long-tailed distribution $F$ is called $\phi$-insensitive (or $\phi$-flat) if $\bar{F}(x \pm \phi(x)) \sim \bar{F}(x)$ as $x \rightarrow \infty$.

Lemma 5.4. For any integrable function $\zeta(y)$ we assume that

$$
\lim _{u \rightarrow \infty} \frac{\int_{\phi(u)}^{\infty} \zeta(y) d y}{\bar{F}_{h}^{e}(u)}=0
$$

where $F_{h}^{e}$ is $\phi$-insensitive. Then it holds that

$$
\lim _{u \rightarrow \infty} \frac{\int_{0}^{u} \bar{F}_{h}^{e}(u-y) \zeta(y) d y}{\bar{F}_{h}^{e}(u)}=\int_{0}^{\infty} \zeta(y) d y<\infty .
$$

Proof. We know from [12, Theorem 2.28, p.23] that $F_{h}^{e}(x)$ is also long-tailed and we can then write for any function $\phi$ such that $F_{h}^{e}$ is $\phi$-insensitive,

$$
\begin{aligned}
\frac{\int_{0}^{u} \bar{F}_{h}^{e}(u-y) \zeta(y) d y}{\bar{F}_{h}^{e}(u)} & =\int_{0}^{\phi(u)} \frac{\bar{F}_{h}^{e}(u-y)}{\bar{F}_{h}^{e}(u)} \zeta(y) d y+\int_{\phi(u)}^{u} \frac{\bar{F}_{h}^{e}(u-y)}{\bar{F}_{h}^{e}(u)} \zeta(y) d y \\
& \leq \int_{0}^{\phi(u)} \frac{\bar{F}_{h}^{e}(u-\phi(u))}{\bar{F}_{h}^{e}(u)} \zeta(y) d y+\int_{\phi(u)}^{u} \frac{\bar{F}_{h}^{e}(u-y)}{\bar{F}_{h}^{e}(u)} \zeta(y) d y .
\end{aligned}
$$

For fixed $y$, it holds by the definition of long-tailed distributions that $\lim _{u \rightarrow \infty} \bar{F}_{h}^{e}(u-y) / \bar{F}_{h}^{e}(u)=1$. Moreover, $\lim _{u \rightarrow \infty} \bar{F}_{h}^{e}(u-\phi(u)) / \bar{F}_{h}^{e}(u)=1$ by virtue of $\phi$-insensitivity. Consequently, for $u$ large enough, there exist small $\epsilon_{1}, \epsilon_{2}>0$ such that $\bar{F}_{h}^{e}(u-\phi(u)) / \bar{F}_{h}^{e}(u) \leq 1+\epsilon_{1}$. Moreover, $\int_{\phi(u)}^{u} \frac{\bar{F}_{h}^{e}(u-y)}{\bar{F}_{h}^{e}(u)} \zeta(y) d y \leq \int_{\phi(u)}^{\infty} \zeta(y) d y / \bar{F}_{h}^{e}(u)$, which tends to zero by (45), hence can be bounded by some $\epsilon_{2}$ for sufficiently large $u$. Thus, we can write using Equation (46) that

$$
\lim _{u \rightarrow \infty} \frac{\int_{0}^{u} \bar{F}_{h}^{e}(u-y) \zeta(y) d y}{\bar{F}_{h}^{e}(u)} \leq\left(1+\epsilon_{1}\right) \int_{0}^{\phi(u)} \zeta(y) d y+\epsilon_{2} \leq\left(1+\epsilon_{1}\right) \int_{0}^{\infty} \zeta(y) d y+\epsilon_{2},
$$

where we used that $\zeta(y)$ is integrable. The required result now follows by letting $\max \left\{\epsilon_{1}, \epsilon_{2}\right\} \rightarrow 0$.

Theorem 5.5. When $\omega$ is a bounded function with $\omega \leq a$, we have

$$
\lim _{u \rightarrow \infty} \frac{\widehat{G S_{\omega}^{\epsilon}}(u, q)}{\bar{F}_{h}^{e}(u)} \leq \epsilon A(q) \lambda^{(-)}\left(a \mu_{h}\left(\mathbf{W}^{(q)}(0)\right)_{(1,1)}+\int_{0}^{\infty} \kappa(x) d x\right),
$$

where

$$
\begin{aligned}
\kappa(x)= & -\lambda^{(-)} \mu_{h}\left(\mathbf{W}^{(q)}(d x)\right)_{(1,1)} *\left(\mathbf{W}^{(q)}(d x)\right)_{1 \bullet} \cdot \int_{0}^{\infty} \int_{0}^{\infty} \omega(y, z)\left(e^{-\mathbf{R}^{(q)} z}\right)_{\bullet 1} F_{p}(z+d y) d z \\
& +\lambda^{(-)} \mu_{h}\left(\mathbf{W}^{(q)}(d x)\right)_{(1,1)}^{* 2} \int_{0}^{\infty} \int_{0}^{x} \omega(y, z) F_{p}(z+d y) d z+a \mu_{h}\left(\mathbf{W}^{(q)}(d x)\right)_{(1,1)} .
\end{aligned}
$$

The equality holds exactly when $\omega \equiv a$.

Proof. The terms of $\widehat{G S_{\omega}^{\epsilon}}(u, q)$ that are bounded by an exponential vanish in the tail and the asymptotic behavior of the approximation can only be attributed to the terms involving $F_{h}^{e}$. For the latter terms, it suffices 
to note that the function $\kappa(x)$ is integrable and satisfies (45) due to (41) (since $\bar{F}_{h}^{e}(z)$ is heavy-tailed) and apply Lemma 5.4. The integrability of $\kappa(x)$ comes from the integrability of the scale matrix $\mathbf{W}^{(q)}(d x)$, which can be proven by using similar arguments as in Lemma 5.3 .

\section{Conclusions}

This work complements other investigations of the Gerber-Shiu function for risk processes with two-sided jumps; see e.g. [1, 20, 24]. However, they all assume special distributions with light tails for the claim sizes. In contrast, our paper allows for additional heavy-tailed claims that may appear with a small probability.

Specifically, we combined perturbation analysis with fluid embedding to construct approximations for the Gerber-Shiu function. The developed approximations have a proven $O\left(\epsilon^{2}\right)$ error and a heavy-tailed behavior in the tail. Moreover, the derived closed-form formulas are not only suitable to produce numerical estimates for the GS function but allow us additionally to study theoretical properties of the approximation. Moreover, it could be feasible to calculate exact tail asymptotics when all involved quantities are defined explicitly.

Note that one possible generalization is related with the addition of an independent Brownian component to the risk process. However, the analysis becomes much more complex since Brownian motion could cause ruin by creeping and this case is not treated by our approach. Finally, another possible extension that needs to be researched by its own is to construct approximations for the GS function of a general MAP with all upward jumps of phase-type.

\section{Acknowledgements}

The work of Zbigniew Palmowski is partially supported by National Science Centre Grant No. 2016/23/B/HS4/00566 (2017-2020). Eleni Vatamidou acknowledges financial support from the Swiss National Science Foundation Project 200021_168993.

\section{References}

[1] H. Albrecher, H. U. Gerber, and H. Yang. "A direct approach to the discounted penalty function". In: North American Actuarial Journal 14.4 (2010), pp. 420-434.

[2] S. Asmussen and H. Albrecher. Ruin Probabilities. Second. Advanced Series on Statistical Science \& Applied Probability, 14. World Scientific, 2010.

[3] S. Asmussen, F. Avram, and M. R. Pistorius. "Russian and American Put Options Under Exponential Phase-Type Lévy Models". In: Stochastic Processes and their Applications 109.1 (2004), pp. 79-111.

[4] A. L. Badescu, L. Breuer, S. Drekic, G. Latouche, and D. A. Stanford. "The surplus prior to ruin and the deficit at ruin for a correlated risk process". In: Scandinavian Actuarial Journal 2005.6 (2005), pp. 433445 .

[5] E. Biffis and A. E. Kyprianou. "A note on scale functions and the time value of ruin for Lévy insurance risk processes". In: Insurance: Mathematics \& Economics 46.1 (2010), pp. 85-91.

[6] E. Biffis and M. Morales. "On a generalization of the Gerber-Shiu function to pathdependent penalties". In: Insurance: Mathematics \& Economics 46.1 (2010), pp. 92-97.

[7] L. Breuer and A. L. Badescu. "A generalised Gerber-Shiu measure for Markov-additive risk processes with phase-type claims and capital injections". In: Scandinavian Actuarial Journal 2014.2 (2014), pp. 93-115.

[8] S. Chiu and C. Yin. "The time of ruin, the surplus prior to ruin and the deficit at ruin for the classical risk process perturbed by diffusion". In: Insurance: Mathematics and Economics 33.1 (2003), pp. 59-66. 
[9] I. Czarna, A. Kaszubowski, S. Li, and Z. Palmowski. "Fluctuation identities for omega-killed Markov additive processes and dividend problem". In: arXiv preprint arXiv:1806.08102 (2018).

[10] P. Embrechts, C. Klüppelberg, and T. Mikosch. Modelling Extremal Events: for Insurance and Finance. Vol. 33. Applications of Mathematics. Springer-Verlag, 1997.

[11] R. Feng and Y. Shimizu. "Potential measures for spectrally negative Markov additive processes with applications in ruin theory". In: Insurance: Mathematics $\&$ Economics 59 (2014), pp. 11-26.

[12] S. Foss, D. Korshunov, S. Zachary, et al. An Introduction to Heavy-Tailed and Subexponential Distributions. 2nd. Vol. 6. Springer, 2011.

[13] H. U. Gerber and E. S. W. Shiu. "On the time value of ruin". In: North American Actuarial Journal 2.1 (1998), pp. 48-78.

[14] H. U. Gerber and E. S. W. Shiu. "The time value of ruin in a Sparre Andersen model". In: North American Actuarial Journal 9.2 (2005), pp. 49-54.

[15] D. Hua and L. Zaiming. "The ruin problem in a renewal risk model with two sided jumps". In: Mathematical and Computer Modeling 57.3-4 (2013), pp. 800-811.

[16] J. Ivanovs. "One-sided Markov Additive Processes and Related Exit Problems". PhD thesis. Universiteit van Amsterdam, 2011.

[17] J. Ivanovs. "Potential measures of one-sided Markov additive processes with reflecting and terminating barriers". In: Journal of Applied Probability 51.4 (2014), pp. 1154-1170.

[18] J. Ivanovs and Z. Palmowski. "Occupation densities in solving exit problems for Markov additive processes and their reflections". In: Stochastic Processes and their Applications 122.9 (2012), pp. 3342-3360.

[19] M. Jacobsen. "The time to ruin for a class of Markov additive risk process with two-sided jumps". In: Advances in Applied Probability 37.4 (2005), pp. 963-992.

[20] L. Ji and C. Zhang. "The Gerber-Shiu penalty functions for two classes of renewal risk processes". In: Journal of Computational and Applied Mathematics 233.10 (2010), pp. 2575-2589.

[21] T. Kato. Perturbation Theory for Linear Operators. Corrected Printing of the 2nd Edition of 1980. Berlin: Springer-Verlag, 1995.

[22] E. T. Kolkovska and E. M. M. González. Gerber-Shiu functionals for two-sided jumps risk processes perturbed by an $\alpha$-stable motion. 2015.

[23] A. E. Kyprianou. Gerber-Shiu risk theory. Springer Science \& Business Media, 2013.

[24] C. Labbé and K. P. Sendova. "The expected discounted penalty function under a risk model with stochastic income". In: Applied Mathematics and Computation 215.5 (2009), pp. 1852-1867.

[25] S. J. Li and J. Garrido. "The Gerber-Shiu function in a Sparre Andersen risk process perturbed by diffusion". In: Scandinavian Actuarial Journal 2005.3 (2005), pp. 161-186.

[26] Y. Lu and C. C.-L. Tsai. "The expected discounted penalty at ruin for a Markov-modulated risk process perturbed by diffusion". In: North American Actuarial Journal 11.2 (2007), pp. 136-152.

[27] M. Olvera-Cravioto, J. Blanchet, and P. Glynn. "On the transition from heavy traffic to heavy tails for the $M / G / 1$ queue: the regularly varying case". In: The Annals of Applied Probability 21.2 (2011), pp. 645-668.

[28] E. Vatamidou. "Error analysis of stuctured Markov chains". PhD thesis. Eindhoven University of Tehcnology, 2015.

[29] E. Vatamidou, I. J.B. F. Adan, M. Vlasiou, and B. Zwart. "Corrected phase-type approximations of heavytailed risk models using perturbation analysis". In: Insurance: Mathematics \& Economics 53.2 (2013), pp. 366-378. 
[30] X. Xing, W. Zhang, and Y. Jiang. "On the time to ruin and the deficit at ruin in a risk model with double-sided jumps". In: Statistics \& Probability Letters 78.16 (2008), pp. 2692-2699.

[31] Z. Zhang, H. Yang, and S. Li. "The perturbed compound Poisson risk model with two-sided jumps". In: Journal of Computational and Applied Mathematics 233.8 (2010), pp. 1773-1784. 